\title{
Surface-assembled poly(l:C) on PEGylated PLGA microspheres as vaccine adjuvant: APC activation and bystander cell stimulation ${ }^{\text {is }}$
}

Annina M. Hafner ${ }^{a}$, Blaise Corthésy ${ }^{b}$, Marcus Textor ${ }^{c}$, Hans P. Merkle ${ }^{a, *}$

Keywords: Immunostimulants; Adjuvants; Poly(I:C); Dendritic cells; Fibroblasts; Microspheres; Vaccine formulation

\begin{abstract}
Abbreviations
APC, antigen presenting cell; CCL21, chemokine (C-C motif) ligand 21; CCR7, chemokine (C-C motif) receptor 7; CBA, cytometric bead array; FSC, forward scattering (measured by flow cytometry) GM-CSF, granulocyte macrophage colony-stimulating factor; HFF, human foreskin fibroblast; IL, interleukin; LPS, lipopolysaccharide; MFI, mean fluorescence intensity; MHC, major histocompatibility complex; MoDC, monocyte-derived dendritic cell; MS, microsphere; PEG, poly(ethylene glycol); PLGA, poly(lactic-co-glycolic acid); PLGA(tt), PLGA microsphere formulation loaded with tetanus toxoid; PLL, poly(L-lysine); PLL-g-PEG, poly(L-lysine)-graft-poly(ethylene glycol); poly(I:C), polyriboinosinic acid-polyribocytidylic acid; PS, polystyrene; SSC, side scattering (measured by flow cytometry); TLR, Toll-like receptor; TNF- $\alpha$, tumor necrosis factor- $\alpha$; tt, tetanus toxoid
\end{abstract}




\begin{abstract}
Biodegradable poly(lactic-co-glycolic acid) (PLGA) microspheres are potential vehicles to deliver antigens for vaccination. Because they lack the full capacity to activate professional antigen presenting cells (APCs), combination with an immunostimulatory adjuvant may be considered. A candidate is the synthetic TLR3 ligand polyriboinosinic acid-polyribocytidylic acid, poly(I:C), which drives cell-mediated immunity. However, poly(I:C) has also been linked to the pathogenesis of autoimmunity, as affected by widespread stimulation of nonhematopoietic bystander cells. To address this aspect, we propose to minimize the poly(l:C) dose as well as to control the stimulation of non-immune bystander cells by poly(l:C). To facilitate the maturation of APCs with minimal poly $(\mathrm{I}: \mathrm{C})$ doses, we surface-assembled poly(I:C) onto PLGA microspheres. The microspheres' surface was further modified by poly(ethylene glycol) (PEG) coronas with varying PEG-densities. PLGA microspheres loaded with tetanus toxoid (tt) as model antigen were manufactured by microextrusion-based solvent extraction. The negatively charged PLGA(tt) microspheres were coated with polycationic poly(L-lysine) (PLL) polymers, either PLL itself or PEG-grafted PLL (PLL-g-PEG) with varying grafting ratios $(g=2.2$ and $g=10.1)$. Stable surface assembly of poly(I:C) was achieved by subsequent incubation of polymer-coated PLGA microspheres with aqueous poly(I:C) solutions. We evaluated the immunostimulatory potential of such PLGA(tt) microsphere formulations on monocyte-derived dendritic cells (MoDCs) as well as human foreskin fibroblasts (HFFs) as model for non-hematopoietic bystander cells. Formulations with surface-assembled poly $(\mathrm{I}: \mathrm{C})$ readily activated MoDCs with respect to the expression of maturation-related surface markers, proinflammatory cytokine secretion and directed migration. When surface-assembled, poly $(\mathrm{I}: \mathrm{C})$ enhanced its immunostimulatory activity by more than one order of magnitude as compared to free poly $(\mathrm{I}: \mathrm{C})$. On fibroblasts, surface-assembled poly $(\mathrm{I}: \mathrm{C})$ upregulated class I MHC but not class II MHC. Phagocytosis of PLGA(tt) microsphere formulations by MoDCs and HFFs remained mostly unaffected by PEG-grafted PLL coatings. In contrast, high concentrations of free poly(l:C) led to a marked drop of microsphere phagocytosis by HFFs. Overall, surface assembly on PEGylated PLGA microspheres holds promise to improve both efficacy and safety of poly $(\mathrm{I}: \mathrm{C})$ as vaccine adjuvant.
\end{abstract}




\section{Introduction}

Biodegradable poly(lactic-co-glycolic acid) (PLGA) microspheres represent an established vehicle for drug and vaccine delivery with a documented safety record (Langer et al., 1997; Johansen et al., 2000). In the optimal particle size range of about 1 - $10 \mu \mathrm{m}$ PLGA microspheres are readily phagocytosed by professional antigen presenting cells (APCs), such as dendritic cells (DCs) and macrophages. Previous studies showed that microencapsulation of an antigen in PLGA microspheres reduced the antigen load necessary to achieve immunity, markedly prolonged antigen presentation and favoured cross-presentation of the encapsulated antigen (Men et al., 1997; Evans et al., 2004; Waeckerle-Men et al., 2005). A comprehensive review on the immunogenicity of PLGA particulates for the delivery of subunit vaccines has been recently published (Silva et al., 2016). Nevertheless, PLGA microspheres alone lack the full capacity to activate APCs to induce a potent immune response. Hence, complementary combination with an immunostimulatory adjuvant may be promising. To this end, formulation development has been claimed to play a key role (O'Hagan and Fox, 2015).

A promising immunostimulant is the synthetic TLR3 ligand polyriboinosinic acidpolyribocytidylic acid, poly(I:C) (Jin et al., 2010; Salvador et al., 2012; Hafner et al., 2013; Martins et al., 2014; Ammi et al., 2015). It is a mimic of viral dsRNA which drives cellmediated immunity. On the down side, poly $(\mathrm{I}: \mathrm{C})$ has also been linked to the pathogenesis of autoimmunity, as affected by a wide spread stimulation of non-hematopoietic bystander cells (Lang et al., 2015). For instance, an aberrant class II major histocompatibility complex (MHC) molecule expression was reported in a variety of non-hematopoietic cells after the translocation of poly $(\mathrm{l}: \mathrm{C})$ into the cytoplasm by means of the transfection reagent lipofectamine (Suzuki et al., 1999). Such an upregulation of class II MHC molecule expression was associated with the danger of developing autoimmune diseases (Bottazzo et al., 1985; Jackson et al., 1988; Kohn et al., 2000). Furthermore, parenteral administration of free poly $(I: C)$ in mice and rats has been identified as a trigger towards autoimmunity (Ewel et al., 1992; Sobel et al., 1992; Okada et al., 2005; Patole et al., 2005). To address this safety aspect we propose to minimize the dose of poly $(\mathrm{I}: \mathrm{C})$ necessary for the activation of APCs, as well as to control the adverse stimulation of non-hematopoietic bystander cells.

For more than a decade the coating of negatively charged PLGA microspheres with cationic polymers as well as the subsequent surface assembly of DNA or RNA were explored for gene as well as nucleic acid adjuvant delivery (Denis-Mize et al., 2000; Singh et al., 2000; Singh et al., 2001; Walter and Merkle, 2002; Jilek et al., 2005; Wischke et al., 2009). Furthermore, surface assembly of poly( $\mathrm{I}: \mathrm{C})$ onto microspheres was reported to result in a 
largely potentiated efficacy to induce maturation of monocyte-derived dendritic cells (MoDCs) as compared to free poly(l:C), allowing to minimize doses (Wischke et al., 2009; Hafner et al., 2011).

Dermal fibroblasts represent an abundant non-hematopoietic bystander cell type at the injection site of a subcutaneously injected vaccine. They express a wide array of pattern recognition receptors (PRRs) including TLR3 and play an important role in stimulating and modulating the response of the innate immune system to invading pathogens (Matsumoto et al., 2002; Ospelt et al., 2008). Thus human foreskin fibroblasts (HFFs) represent a meaningful in vitro model for evaluating their activation by poly(I:C). In a proof-of-concept study with negatively charged carboxylated polystyrene (PS) microspheres of $5 \mu \mathrm{m}$ diameter we demonstrated the potential of poly(ethylene glycol) (PEG) coronas to impede the activation of HFFs by surface-assembled poly(I:C), as reflected by restrained IL-6 secretion and MHC I expression (Hafner et al., 2012). PEG coronas were formed via coating of PS microspheres with polycationic polymers via electrostatic interactions, using a small library of poly(L-lysine)-graft-poly(ethylene glycol) (PLL-g-PEG) copolymers with various degrees of PEGylation. PEGylated surfaces efficiently protected microspheres from aggregation and clotting.

As an expansion of our previous studies (Hafner et al., 2011; Hafner et al., 2012) here we report on PLGA microspheres loaded with encapsulated tetanus toxoid (tt) as model antigen, further on denoted as PLGA(tt), $t \mathrm{t}$ being a frequently applied model antigen for studies with PLGA microparticles, both in vitro and in vivo (Men et al., 1995; Umetsu et al., 1985; Audran et al., 1998; Men et al., 1999; Johansen et al., 2000; Waeckerle-Men et al., 2006). PLGA(tt) microspheres were manufactured by microextrusion-based solvent extraction, using a static micromixer as previously introduced by our group (Freitas et al., 2005a; Freitas et al. 2005b). The resulting negatively charged PLGA(tt) microspheres were either coated with PLL or with one of two PLL-g-PEG copolymers, PLL-10.1-PEG and PLL-2.2-PEG, respectively. Surface assembly of poly $(\mathrm{l}: \mathrm{C})$ was by electrostatically driven adsorption through exposure of the coated PLGA(tt) microspheres to an aqueous poly(l:C) solution.

We evaluated the immunostimulatory potential of such formulations on MoDCs as well as human foreskin fibroblasts (HFFs) as a model for non-hemopoietic bystander cells. The focus for MoDCs was on (i) microsphere phagocytosis, (ii) expression of maturation-related surface markers, (iii) cytokine secretion pattern and (iv) migration capacity of MoDCs after phagocytosis of PLGA(tt) microspheres loaded with surface-assembled poly(I:C). HFFs were studied for microsphere phagocytosis, and for class I and class II MHC molecule expression. 
Surface-assembly of poly(l:C) allowed a dose reduction by more than one order of magnitude as compared to free poly $(\mathrm{I}: \mathrm{C})$. The formulations readily activated MoDCs with respect to the expression of maturation-related surface markers, proinflammatory cytokine secretion as well as directed migration. Phagocytosis of the PLGA(tt) microsphere formulations by MoDCs remained mostly unaffected. In contrast, phagocytosis by HFFs was markedly reduced by free poly(I:C), and upregulation of MHC molecules only occurred for class I but not for class II MHC. Taken together, surface assembly on PEGylated PLGA microspheres holds promise to improve both efficacy and safety of poly $(\mathrm{I}: \mathrm{C})$ as a vaccine adjuvant.

\section{Materials and methods}

\subsection{Polymers}

PLL (20 kDa, as hydrobromide salt; Sigma-Aldrich, Buchs, Switzerland); Cy3-labeled PLL (PLL-Cy3): Fluorescently labelled PLL of $20 \mathrm{kDa}$ was made by conjugation of PLL with the orange fluorescing cyanine dye, Сy3 (ex/em $552 \mathrm{~nm} / 570 \mathrm{~nm}$ ), using the Cy3 mono-reactive dye pack (GE Healthcare Europe, Germany) according to an optimized manufacturer's protocol. In brief, $5 \mathrm{mg}$ PLL were dissolved in $0.5 \mathrm{ml}$ RNA grade $0.1 \mathrm{M} \mathrm{Na}_{2} \mathrm{CO}_{3}(\mathrm{pH}$ 9.3; Sigma-Aldrich, Buchs, Switzerland), sterile filtered and added to the dye vial provided by the manufacturer. Dye and protein solution were thoroughly mixed and incubated during $2 \mathrm{~h}$ at RT under additional mixing every $10 \mathrm{~min}$. Cy3-labelled PLL was separated from excess unconjugated dye using an Amicon Ultra PL-10 centrifugal filter device (Millipore, Zug, Switzerland) with a nominal molecular weight limit of $10 \mathrm{kDa}$, according to the manufacturer's protocol, using sterile-filtered RNA grade 10 mM HEPES buffer $(\mathrm{pH} 7.4$; HEPES buffer) as washing buffer. The purified Cy3-labeled PLL was sterile filtrated and stored at $-24{ }^{\circ} \mathrm{C}$ at a concentration of $1 \mathrm{mg} \mathrm{ml}^{-1}$ in HEPES buffer.

The PLL-g-PEG copolymers used in this study were synthesized as previously outlined (Pasche et al., 2003) and were kindly provided by the Laboratory for Surface Science and Technology of ETH Zurich. Copolymers consisted of a PLL backbone of $20 \mathrm{kDa}$ that was grafted with PEG side chains of $2 \mathrm{kDa}$. Grafting ratios $g$ were 10.1 and 2.2, denoting the ratio of L-lysine units per PEG side chain. The high grafting ratio indicates an open ("mushroom") PEG chain architecture, whereas the low grafting ratio stands for densely packed ("brush") PEG side chains. All polymers were dissolved in HEPES buffer at a concentration of $1 \mathrm{mg} \mathrm{ml}^{-1}$, sterile filtrated and stored at $-24^{\circ} \mathrm{C}$ until needed.

All chemicals used in this study were of analytical grade if not indicated otherwise. Ultrapure water (NANOpure Diamond, Skan AG, Allschwil, Schwitzerland) treated with $0.1 \%$ diethyl 
pyrocarbonate (Sigma-Aldrich, Buchs, Switzerland) was used for RNA grade buffer preparation. Unless otherwise specified all reagents were RNA grade and sterile filtered.

\subsection{Preparation and characterization of PLGA(tt) microspheres}

\subsubsection{Preparation of PLGA(tt) microspheres by microextrusion-based solvent extraction}

Reagents: End group uncapped poly(lactic-co-glycolic acid) (50:50) polymer (PLGA; Resomer $503 \mathrm{H}$; Boehringer Ingelheim, Ingelheim, Germany), tetanus toxoid (tt; Novartis Vaccines and Diagnostics GmbH \& Co., Marburg, Germany), dichloromethane synthesis grade (DCM; Sigma-Aldrich, Buchs, Switzerland), poly(vinyl alcohol) (PVA; Sigma-Aldrich). PLGA(tt) microspheres were prepared by microextrusion-based solvent extraction using a static micromixer (IMM, Mainz, Germany) as previously introduced by us (Freitas et al., $2005 \mathrm{~b}$ ). In brief, $0.8 \mathrm{ml}$ of an aqueous solution containing $2.16 \mathrm{mg} t \mathrm{tt}$ was emulsified into a $5 \% \mathrm{w} / \mathrm{w}$ solution of PLGA in DCM to form a w/o emulsion $(1: 20 \mathrm{w} / \mathrm{w})$. For microsphere preparation the PLGA/tt emulsion and ultrapure water containing $0.5 \% \mathrm{w} / \mathrm{w}$ PVA as aqueous extraction phase were individually fed into the two inlets of the micromixer. The resulting microspheres were collected in a $0.5 \% \mathrm{w} / \mathrm{w}$ aqueous solution of PVA. The dispersion was placed under a laminar air flow for 30 min under stirring, allowing the microspheres to harden and the solvent to evaporate. Separation of hardened microspheres was by filtration through a cellulose membrane ( $0.2 \mu \mathrm{m}$ pore size; GE Healthcare Europe, Germany). Microspheres were dried at 20 mbar at room temperature (RT) and stored desiccated at $4{ }^{\circ} \mathrm{C}$. Encapsulation efficiency for $\mathrm{tt}$ was $61 \%(\mathrm{n}=2)$. Prior to subsequent experiments microspheres were dispersed in HEPES buffer at $2.5 \mathrm{mg} \mathrm{ml}^{-1}$ by ultrasonication (UP $200 \mathrm{H}$ : amplitude 60\%, $0.5 \mathrm{~s}^{-1}, 10$ cycles; Digitana, Lausanne, Switzerland). All preparation steps were carefully conducted under RNase free conditions. Particle size distributions of microspheres were measured by laser light scattering using a Mastersizer X (Malvern Instruments, Worcestershire, UK). The volume mean diameter $D(4,3)$ was $4.1 \mu \mathrm{m}(\mathrm{n}=2)$ with a wide size distribution, $D(v, 0.1)=1.7 \mu \mathrm{m}$ and $\mathrm{D}(\mathrm{v}, 0.9)=7.1 \mu \mathrm{m}$.

\subsubsection{Coating of PLGA(tt) microspheres and surface assembly of poly(l:C)}

Coating of PLGA(tt) microspheres with PLL and with PLL-g-PEG copolymers was with a post-process protocol. Briefly, PLGA(tt) microspheres were dispersed in HEPES buffer $(10 \mathrm{mM}, \mathrm{pH} 7.4, \mathrm{RT})$ at $2.5 \mu \mathrm{g} \mathrm{ml}^{-1}$. The microsphere suspension was mixed at equal volumes of the respective PLL or PLL-g-PEG polymer solution (1 $\mathrm{mg} \mathrm{ml}^{-1}$ in HEPES buffer) and incubated for $30 \mathrm{~min}$ under gentle shaking $\left(500 \mathrm{~min}^{-1}, \mathrm{RT}\right)$. Coated microspheres were 
centrifuged (2000 rcf, 2 min, RT), and washed twice in HEPES buffer. Coated microspheres were resuspended at $2.5 \mathrm{\mu g} \mathrm{ml}^{-1}$ in HEPES buffer. Care was taken to conduct coating under aseptic and RNase-free conditions.

For surface assembly of poly $(\mathrm{l}: \mathrm{C}$ ) we used two batches of poly $(\mathrm{l}: \mathrm{C})$ (GE Healthcare Europe, Germany and Invivogen, San Diego, CA). Poly(I:C) was resuspended in HEPES buffer and stored at $-80^{\circ} \mathrm{C}$. Prior to each experiment poly $(\mathrm{I}: \mathrm{C})$ was rehybridized to ensure doublestranded conformation and the concentration was adjusted to $100 \mathrm{ng} \mathrm{\mu l}^{-1}$. PLL- and PLL-g-PEG-coated microspheres were incubated with $1 \% \mathrm{w} / \mathrm{w}$ poly $(\mathrm{I}: \mathrm{C})$ under gentle shaking (30 min, $500 \mathrm{~min}^{-1}, \mathrm{RT}$ ). For MoDC studies the formulations were directly added to the MoDC cultures. Because of the high sensitivity of HFFs to detect poly(I:C) the formulations were centrifuged (2000 rcf, 2 min, RT), washed two times with HEPES buffer to remove free poly(l:C) and suspended at $2.5 \mathrm{\mu g} \mathrm{ml}^{-1}$ in HEPES buffer. Surface assembly was conducted under aseptic and RNase-free conditions. For yet unknown reasons the direct assessment of the amounts of surface-assembled poly(l:C) on PLGA(tt) using a standard PicoGreen assay led to erroneous results, in contrast to previous studies with PS microspheres. Hence, all dosages of surface assembled poly(I:C) on PLGA(tt) refer to the total amounts introduced for surface assembly.

\subsection{3. $\zeta$-potential measurement of microspheres}

Uncoated, PLL- and PLL-g-PEG-coated microspheres with and without surface-assembled poly(l:C) were dispersed at $50 \mathrm{\mu g} \mathrm{ml}^{-1}$ in HEPES buffer. $\zeta$-potentials were measured with a Malvern Zetasizer 3000 HAS (Malvern Instruments, Worcestershire, UK). Measurements were repeated five times per sample.

\subsection{Cell culture}

\subsubsection{Generation and characterization of MoDCs}

MoDCs were obtained from human peripheral blood. Peripheral blood mononuclear cells (PBMCs) were isolated from buffy coats (Bloodbank Zurich, Zurich, Switzerland) by density gradient centrifugation of Ficoll-Paque plus (GE Healthcare Bio-sciences AB, Uppsala, Sweden). Collected PBMCs were washed three times with Dulbecco's phosphate-buffered saline (D-PBS) (Invitrogen, Basel, Switzerland) and suspended at a concentration of $1.5 \times 10^{7}$ cells $\mathrm{Il}^{-1}$ in RPMI $1640+\mathrm{L}$-glutamine (Invitrogen, Basel, Switzerland) supplemented with $10 \%$ heat-inactivated human serum (three donors pooled) (Bloodbank Zurich, Zurich Switzerland), $100 \mathrm{U} \mathrm{ml}^{1}$ penicillin and $100 \mathrm{\mu g} \mathrm{ml}^{-1}$ streptomycin (Invitrogen, Basel, 
Switzerland). After $1.5 \mathrm{~h}$ incubation $\left(37^{\circ} \mathrm{C} / 5 \% \mathrm{CO}_{2}\right)$ in culture flasks (Nunc, Wiesbaden, Germany) the non-adherent cells were carefully removed. The adherent cell fraction was gently rinsed tree times with D-PBS and further cultured in RPMI medium supplemented with $5 \%$ heat-inactivated human serum and $100 \mathrm{U} \mathrm{ml}^{-1}$ penicillin and $100 \mathrm{\mu g} \mathrm{ml}^{-1}$ streptomycin in the presence of $50 \mathrm{\mu g} \mathrm{ml}^{-1}$ human interleukin-4 (IL-4) (R\&D Systems Europe Ltd., Abington, $\mathrm{UK}$ ) and $50 \mu \mathrm{g} \mathrm{ml}^{-1}$ of human granulocyte macrophage colony-stimulating factor (GM-CSF) (R\&D Systems Europe Ltd., Abington, UK) to differentiate into MoDCs. This medium is subsequently referred to as MoDC-medium. After 3 days, MoDCs were collected from the culture flasks, pooled, resupplied with 33\% fresh MoDC-medium and reseeded in culture dishes at a concentration of $4 \times 10^{5}$ cells $\mathrm{ml}^{-1}$ with $750 \mu \mathrm{l}$ per dish. MoDCs were validated by flow cytometry (see below) confirming the expression of CD11c (clone B-ly6, BD Pharmingen, Basel, Switzerland) and the absence of the monocyte marker CD14 (clone M5E2, BD Pharmingen), and were considered as $>95 \%$ pure.

Mature MoDCs were generated by treatment with $2.5 \mathrm{\mu g} \mathrm{ml}^{-1}$ LPS (Sigma-Aldrich, Buchs, Switzerland) and $20 \mathrm{\mu g} \mathrm{ml}^{-1}$ poly(l:C), respectively, and were positive for the DC-specific maturation marker CD83. All experiments were carried out between day 6 and day 11 of MoDC culture.

Microsphere assays: MoDCs were treated with $100 \mu \mathrm{g}$ of various microsphere formulations as well as free poly(l:C) or LPS over a period of $72 \mathrm{~h}$ if not indicated otherwise.

Microspheres were not washed but the complete assembly preparation was given directly to MoDCs. To prepare for analysis, MoDCs were put on ice, mechanically removed from the culture dishes and used for further analysis.

\subsubsection{Human foreskin fibroblasts}

HFFs from two individuals were purchased from the European collection of cell cultures (ECACC; Wiltshire, UK) and PromoCell GmbH (Heidelberg, Germany), respectively. Cells were cultured as subconfluent monolayers in $75 \mathrm{~cm}^{2}$ cell culture flasks (TPP, Trasadingen, Switzerland) with Dulbecco's modified Eagle medium (D-MEM) GlutaMAX-I (Invitrogen, Basel, Switzerland) supplemented with 10\% fetal bovine serum (FBS) (Invitrogen, Basel, Switzerland), $100 \mathrm{U} \mathrm{ml}^{-1}$ penicillin and $100 \mathrm{~g} \mathrm{ml}^{-1}$ streptomycin (Invitrogen, Basel, Switzerland) (D-MEM/10\%) at $37{ }^{\circ} \mathrm{C} / 5 \% \mathrm{CO}_{2}$. For passaging (1:5), HFFs were washed with Dulbecco's phosphate-buffered saline (D-PBS; Invitrogen, Basel, Switzerland) and detached with trypsin-EDTA $0.25 \%$ (Invitrogen, Basel, Switzerland) 5 min at $37{ }^{\circ} \mathrm{C} / 5 \% \mathrm{CO}_{2}$. HFFs were seeded at $5 \times 10^{4}$ cells $\mathrm{ml}^{-1}$ in 24 -well culture dishes in $500 \mathrm{ml}$ culture medium per well. 
All assays were performed with confluent HFF layers over a time period of $48 \mathrm{~h}$ and between passages 7 and 14 .

Microsphere assays: HFFs were treated with $100 \mu \mathrm{g}$ of various microsphere formulations as well as free poly(l:C) $\left(0.1 \mathrm{\mu g} \mathrm{ml}^{-1}, 1 \mu \mathrm{g} \mathrm{ml}^{-1}\right.$ and $\left.10 \mu \mathrm{g} \mathrm{ml}^{-1}\right)$ over a period of $48 \mathrm{~h}$. To prepare for analysis, supernatants were removed and HFFs were incubated with $0.25 \%$ EDTA in DPBS for $5 \mathrm{~min}$ at $37{ }^{\circ} \mathrm{C} / 5 \% \mathrm{CO}_{2}$. Subsequently HFFs were put on ice and $1 \mathrm{ml}$ ice cold DMEM/10\% was added. The now feebly attached HFFs were removed from the culture dish by back-and-forth pipetting and used for further analysis.

\subsection{Flow cytometry}

\subsubsection{Expression of surface markers}

MoDCs, HFFs were analyzed by flow cytometry (FACSCanto ${ }^{\mathrm{TM}}$, BD, Franklin Lakes, NJ) after staining with the respective antibodies according to the manufacturer's protocols: CCR7 (clone 150503, R\&D Systems Europe Ltd., Abington, UK), CD80 (clone L307.4), CD83 (clone HB15e), CD86 (clone 2331), HLA-A, B, C (clone G46-2.6) and HLA-DP, DQ, DR (clone Tü39). The respective isotype controls were IgG2a (clone G155-178) and IgG1 (clone MOPC-21). If not indicated otherwise, antibodies were from BD Pharmingen, Basel, Switzerland. Throughout, flow cytometry data was analyzed with FlowJo software (V.7.2.5, Tree Star Inc., Ashland, USA).

\subsubsection{Phagocytosis}

MoDCs were incubated with $100 \mu \mathrm{g}$ of PLL- and PLL-g-PEG-coated as well as fluorescent PLL-Cy3-coated PLGA(tt) microspheres without and with surface-assembled poly(I:C). Cy3fluorescence as well as the mean side scatter (SSC) signal was analyzed by flow cytometry $72 \mathrm{~h}$ after microsphere administration. The mean SSC signal is proportional to cell granularity or internal complexity and can therefore be used as a measure for phagocytosis, as previously established for MoDCs and macrophages (Wattendorf et al., 2006).

HFFs were treated with $100 \mu \mathrm{g}$ of PLL- and PLL-g-PEG-coated microspheres without and with surface-assembled poly(l:C). Phagocytosis was qualitatively analyzed by flow cytometry $48 \mathrm{~h}$ after microsphere administration using the mean SSC signal and Cy3-fluorescence, respectively.

To assess the influence of poly(l:C) on the phagocytic activity of HFFs, HFFs were stimulated with $10 \mathrm{\mu g} \mathrm{ml}^{-1}$ free poly(l:C), either $5 \mathrm{~h}$ prior to or concurrent with the 
administration of $100 \mu \mathrm{g}$ of fluorescent PLL-Cy3-coated PLGA(tt) microspheres. $48 \mathrm{~h}$ after microsphere administration phagocytosis of microspheres by HFFs was analyzed by flow cytometry using the mean SSC signal as well as Cy3-fluorescence. Untreated HFFs were used as background control.

\subsection{Chemotaxis assay}

In order to study the potential of surface-assembled poly $(\mathrm{I}: \mathrm{C})$ to upregulate the chemokine (C-C motif) receptor 7 (CCR7) in MoDCs as well as the capacity of such stimulated MoDCs to migrate along a chemokine ( $\mathrm{C}-\mathrm{C}$ motif) ligand 21 (CCL21) gradient, a ligand of CCR7, we used a chemotaxis Transwell assay. With some adjustments the assay was performed according to a modified protocol used by Waeckerle-Men et al. (2004). Briefly, $48 \mathrm{~h}$ after the respective treatment all MoDCs were mechanically removed from the culture dishes. $10^{5}$ MoDCs were placed in the upper chamber of 24-Transwell plates equipped with a polycarbonate membrane of $5 \mu \mathrm{m}$ pore size (Corning Life Sciences, Lowell, MA) in a total volume of $100 \mu \mathrm{l} .600 \mu \mathrm{l}$ of RPMI medium (5\% heat inactivated human serum, $100 \mathrm{U} \mathrm{ml}^{-1}$ penicillin, $100 \mathrm{\mu g} \mathrm{ml}^{-1}$ streptomycin) alone or conditioned with $250 \mathrm{ng} \mathrm{ml}^{-1}$ recombinant human CCL21 (R\&D Systems Europe Ltd., Abington, UK) were given to the bottom chamber. The plate was incubated for $4 \mathrm{~h}$ at $37^{\circ} \mathrm{C}$ and $5 \% \mathrm{CO}_{2}$. Thereafter the Transwells were carefully removed. Migrated MoDCs were collected from the bottom chamber and counted for $60 \mathrm{~s}$ at maximum flow by flow cytometry.

\subsection{Cytokine analysis by cytometric bead array (CBA)}

Supernatants of untreated MoDCs and MoDCs treated with free or surface-assembled poly (I:C) were collected and stored at $-24{ }^{\circ} \mathrm{C}$ in protein LoBind microcentrifuge tubes (Vaudaux-Eppendorf, Basel, Switzerland). Expression of tumor necrosis factor- $\alpha$ (TNF- $\alpha$ ), interleukin (IL)-6, IL-10 and IL-12p70 was analyzed simultaneously using a cytometric bead array (CBA) (BD Bioscience, Basel, Switzerland) according to the manufacturer's protocol.

\section{Results}

\subsection{Microsphere characterization}

By assessment of the $\zeta$-potential we verified the presence of PLL, PLL-10.1-PEG and PLL-2.2-PEG coatings on PLGA(tt)-microspheres as well as the subsequent surface assembly of poly(l:C). All coatings with cationic polymers inverted the originally negative 
$\zeta$-potential of the PLGA(tt)-microspheres to slightly positive values. Subsequent incubation with poly $(\mathrm{I}: \mathrm{C})$ led to even more negative $\zeta$-potentials than measured with untreated PLGA(tt) microspheres, providing evidence for surface-assembled poly(I:C) (Fig. 1). When coated with unPEGylated cationic polymers and PLL-10.1-PEG, the extent of charge inversion after surface assembly of poly $(\mathrm{I}: \mathrm{C})$ was in the same range. Poly $(\mathrm{I}: \mathrm{C})$ surface assembly on PLL-2.2-PEG-coated microspheres resulted in less negative $\zeta$-potentials, indicating reduced surface assembly of poly $(\mathrm{I}: \mathrm{C})$ as compared to the other two formulations. PEGylated coatings efficiently protected the microspheres from aggregation and clotting.

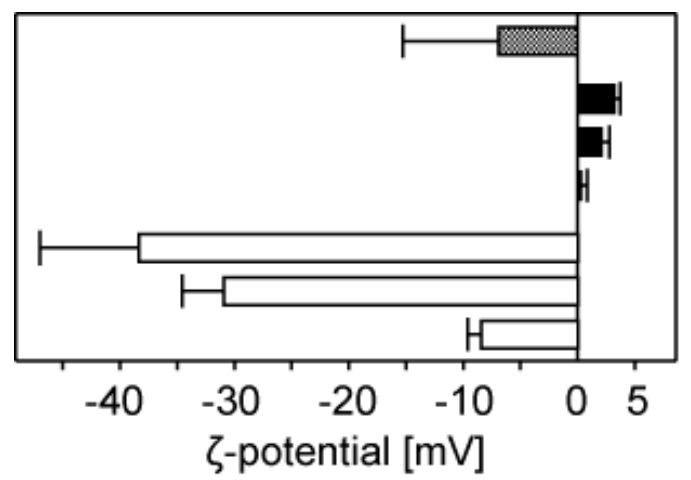

\section{$\operatorname{PLGA}(\mathrm{tt})$}

PLL

PLL-10.1-PEG

PLL-2.2-PEG

PLL-poly(l:C)

PLL-10.1-PEG-poly(I:C)

PLL-2.2-PEG-poly(l:C)

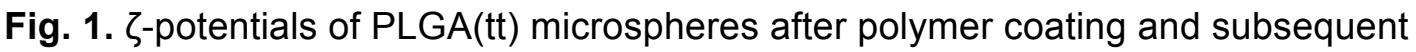
poly $(\mathrm{I}: \mathrm{C})$ surface assembly. $\zeta$-potentials $(\mathrm{mV})$ of uncoated PLGA(tt) (grey bar) as well as PLL-, PLL-10.1-PEG- and PLL-2.2-PEG-coated PLGA(tt) microspheres, prior to (black bars) and post surface assembly of poly(l:C) (open bars). Mean $\pm S D ; n=4$.

\subsection{Microsphere phagocytosis by MoDCs}

Previous experiments with PLL-g-PEG polymer coatings on flat $\mathrm{Nb}_{2} \mathrm{O}_{5}$ surfaces and on PS microspheres suggested that PLL-2.2-PEG coatings result in a densely-packed ("brush") PEG conformation, while PLL-10.1-PEG coatings form an open-structured ("mushroom") one (Pasche et al., 2003). Phagocytosis of PLL-g-PEG-coated PS microspheres with or without surface-assembled poly $(\mathrm{I}: \mathrm{C})$ was found to be exclusively dependent on the density of the formed PEG corona as represented by the grafting ratio $g$ (Wattendorf et al., 2006; Hafner et al., 2011). Here we evaluated whether this also applies to PLL-g-PEG-coated biodegradable PLGA(tt) microspheres.

To assess the phagocytosis of PLGA(tt) microspheres by MoDCs we used the same flow cytometric protocol as previously applied for PS microspheres (Wattendorf et al., 2006; Hafner et al., 2011). In brief, the mean side scatter (SSC) signal of the MoDC population was 
used as a measure for the change in granularity or internal complexity of the cells after phagocytosis of microspheres. MoDCs were incubated for $72 \mathrm{~h}$ with three differently coated PLGA(tt) microsphere formulations either with or without surface-assembled poly(l:C). The coatings were PLL, PLL-10.1-PEG and PLL-2.2-PEG. In order to directly visualize microsphere phagocytosis, MoDCs were also incubated with fluorescent PLL-Cy3-coated PLGA(tt) microspheres with or without surface-assembled poly(I:C). Results are presented in Fig. 2.

A

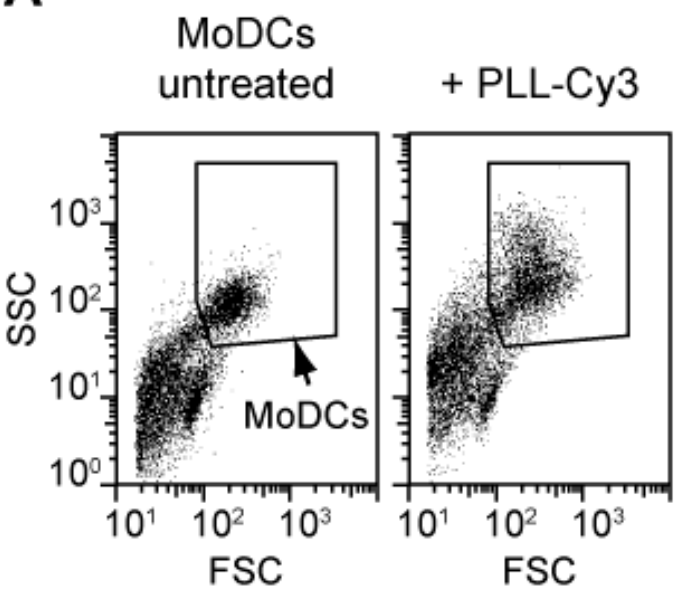

C

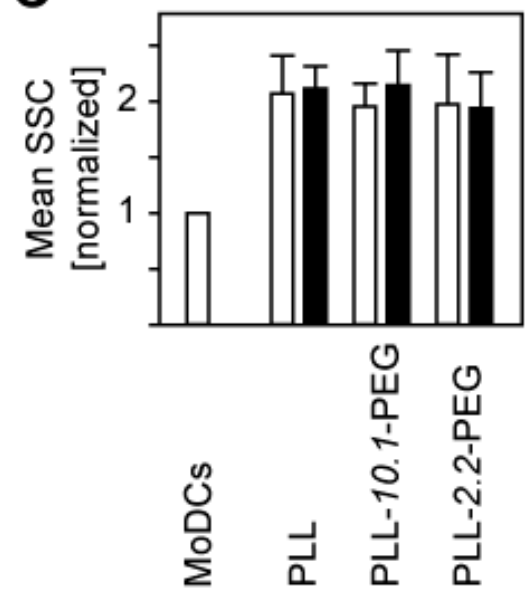

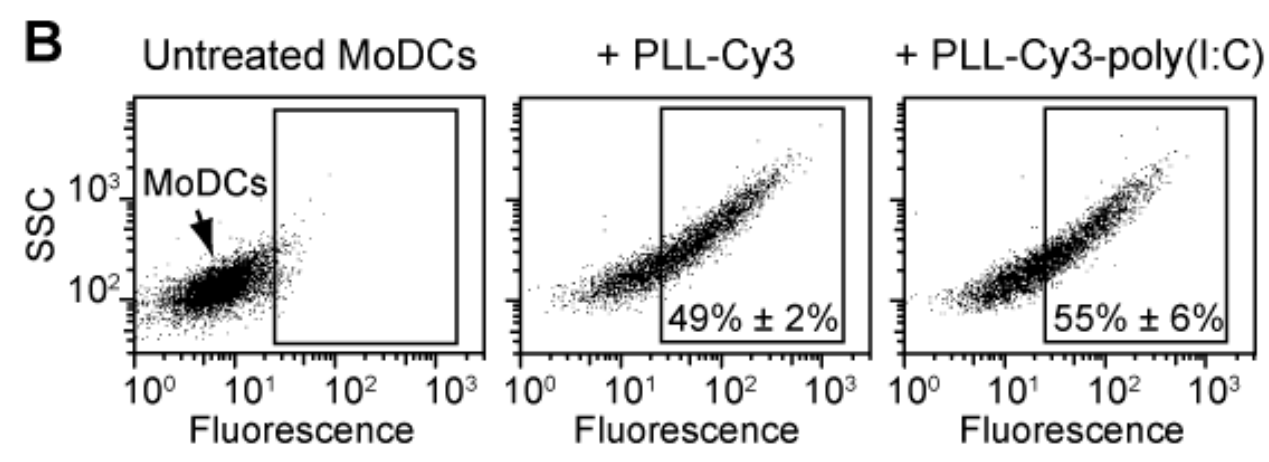

Fig. 2. Phagocytosis of PLGA(tt) microspheres as assessed by flow cytometry. A. Gating of the MoDC cells by their granularity (SSC signal) and size (FSC signal) using the SSC/FSC signal plots. Untreated MoDCs (left panel) and MoDCs $72 \mathrm{~h}$ after incubation with PLL-Cy3coated microspheres. B. MoDCs incubated with fluorescent PLL-Cy3-coated microspheres either without poly(l:C) (middle panel) or with surface-assembled poly(l:C) (right panel). Untreated MoDCs are shown as reference (left panel). Gate encloses MoDCs that have phagocytosed fluorescent microspheres. SSC/fluorescence dot plots of one representative experiment out of three are shown. Numbers given indicate the percentage of MoDCs with phagocytosed fluorescent microspheres. Mean $\pm S D, n=3$. C. Mean SSC signals of 
MoDCs treated for $72 \mathrm{~h}$ with various microsphere formulations: PLGA(tt) microspheres coated with either PLL, PLL-10.1-PEG or PLL-2.2-PEG without poly(I:C) and untreated MoDCs as reference (open bars); PLGA(tt) microspheres coated with either PLL ( $n=9)$, PLL-10.1-PEG ( $n=6)$ or PLL-2.2-PEG ( $n=9)$ and loaded with surface-assembled poly(l:C) (black bars). Data was normalized to untreated MoDCs as reference. Mean \pm SD.

A pronounced shift of the MoDC population towards an increased mean SSC signal as compared to untreated MoDCs is exemplified by the effect of phagocytosed PLL-Cy3-coated microspheres on the granularity of the MoDCs (Fig. 2A). In accordance with the mean SSC signals, phagocytosis of fluorescent PLL-Cy3-coated microspheres, with or without surfaceassembled poly $(\mathrm{I}: \mathrm{C})$, showed no difference, neither as to the mean Cy3-fluorescence intensity of the MoDC populations (MFI $I_{P L L-C y 3}: 55 \pm 10, n=3$; $M_{P I} I_{P L-C y 3-p o l y(I: C)}: 45 \pm 3, n=3$ ), nor in the number of MoDCs with fluorescent PLL-Cy3-coated microspheres (\%PLL-Cy3: $49 \pm 2, n=3$; \% the SSC signal and the Cy3 fluorescence: Increasing SSC values corresponded with increasing Cy3 fluorescence (Fig. 2B), supporting the application of the SSC signal as a tool to monitor the phagocytosis of microspheres by MoDCs. Finally, PLGA(tt) microspheres coated with either PLL, PLL-10.1-PEG or PLL-2.2-PEG were readily phagocytosed by MoDCs. Their normalized mean SSC shifts towards towards 2- to 3-fold higher values as compared to untreated MoDCs as control were in the same range, regardless of the degree of PEGylation and with or without surface-assembled poly(I:C) (Fig. 2C).

\subsection{Enhanced immunostimulatory efficacy of surface-assembled versus free poly(l:C)}

The immunostimulatory potential of surface-assembled poly(l:C) on PLL- and PLL-g-PEGcoated PS microspheres was previously studied by assessing the upregulation of maturation-related surface markers on MoDCs after microsphere phagocytosis. The results indicated a strongly enhanced immunostimulatory efficacy of surface-assembled poly(l:C) as compared to the same dose of free poly(I:C). In addition, the immunostimulatory potential also depended on the grafting ratio of the coating polymer (Hafner et al., 2011). Here we evaluated whether these properties were transferrable to PLGA(tt) microspheres with distinctly different physical characteristics, such as wider particle size distribution and a less negative $\zeta$-potential. As described in the previous paragraph, MoDCs were incubated for $72 \mathrm{~h}$ with three differently coated PLGA(tt) microsphere formulations either with or without surface-assembled poly $(\mathrm{I}: \mathrm{C})$. In order to compare surface-assembled and free poly $(\mathrm{I}: \mathrm{C})$, MoDCs were exposed to free poly $(\mathrm{I}: \mathrm{C})$ in a dose equivalent to that used for surface 
assembly (in total $1 \mu \mathrm{g}$ per $100 \mu \mathrm{g}$ microspheres or $1.3 \mu \mathrm{g} \mathrm{ml}^{-1}$ ). As positive controls for MoDCs maturation, MoDCs were treated with $20 \mathrm{\mu g} \mathrm{ml}^{-1}$ poly(l:C) or LPS. Untreated MoDCs were used as reference for the immature state. The surface expression of the maturation marker CD83, the costimulatory molecules CD80 and CD86, as well as class I and class II MHC molecules were analyzed by flow cytometry. Results are visualized in Fig. 3 (representative histogram plots of fluorescence intensities) and Fig. 4 (normalized MFIs).

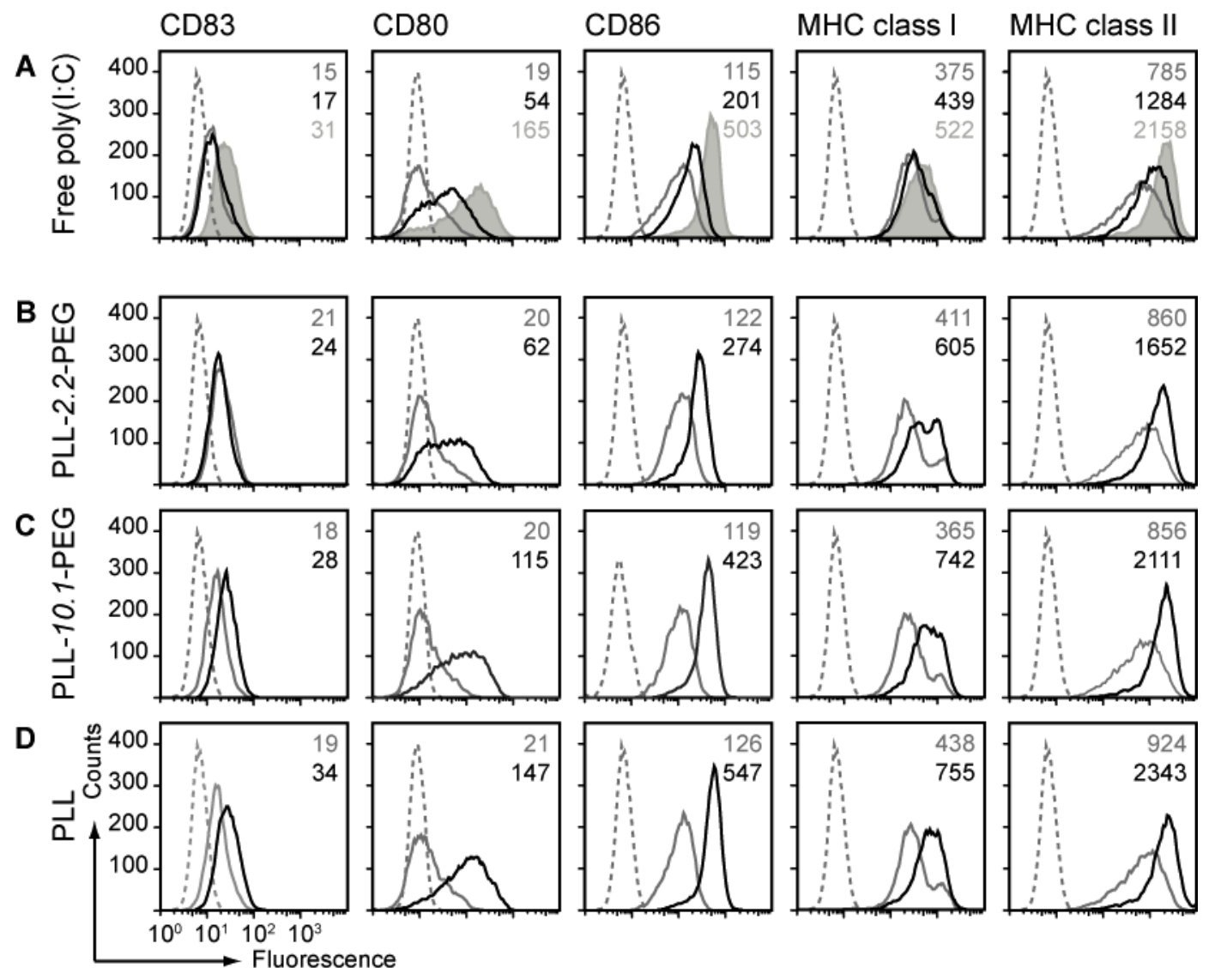

Fig. 3. Histogram plots of maturation-related molecule expression by MoDCs stimulated with surface-assembled poly(l:C) on PLL- or PLL g PEG-coated PLGA(tt) microspheres. One representative experiment out of five is shown. Flow cytometric analyses of the surface expression of CD83, CD80, CD86, class I and class II MHC molecules by MoDCs three days after exposure to: A. $1.3 \mathrm{\mu g} \mathrm{ml}^{-1}$ (black lines) or $20 \mathrm{\mu g} \mathrm{ml}^{-1}$ (light grey peak areas) free poly(I:C); B. $1.3 \mu \mathrm{g} \mathrm{ml} 1$ total poly(I:C) per well for surface assembly on PLL-2.2-PEG-coated PLGA(tt) microspheres; C. on PLL-10.1-PEG-coated PLGA(tt) microspheres; D. on PLLcoated PLGA(tt) microspheres (all black lines). Throughout, isotype controls are given by dotted grey lines, negative controls by grey lines: A. immature MoDCs, B - D. PLL- or PLLg-PEG-coated PLGA(tt) microspheres without poly(I:C). One representative experiment out of five is shown. Numbers in grey, black and light grey indicate the respective MFI values. 
MoDCs readily recognized surface-assembled poly(l:C) on all formulations and responded by upregulation of CD83, CD80, CD86 and class I as well as class II MHC molecule expression. All expression levels, except after exposure to surface-assembled poly(l:C) on PLL-2.2-PEG-coated microspheres, were substantially higher than those obtained with the same dosage of free poly $(I: C)$ and in the same range as the fully mature expression levels obtained with a fifteen times higher dosage of free poly(I:C). Thus, surface-assembled poly(I:C) on PLGA(tt) microspheres was confirmed to display an enhanced immunostimulatory efficacy. Interestingly, when surface-assembled on PLL-2.2-PEG-coated microspheres, poly $(\mathrm{I}: \mathrm{C})$ resulted in a somewhat weaker upregulation of the surface-markers only. Throughout, MoDC exposure to PLGA(tt) microsphere formulations without surfaceassembled poly $(\mathrm{I}: \mathrm{C})$ resulted in expression levels comparable to untreated MoDCs. Hence, the encapsulated tt lacked appreciable effects on surface marker expression. Expectedly, the upregulation of the maturation markers was donor-dependent and varied between the five donors.

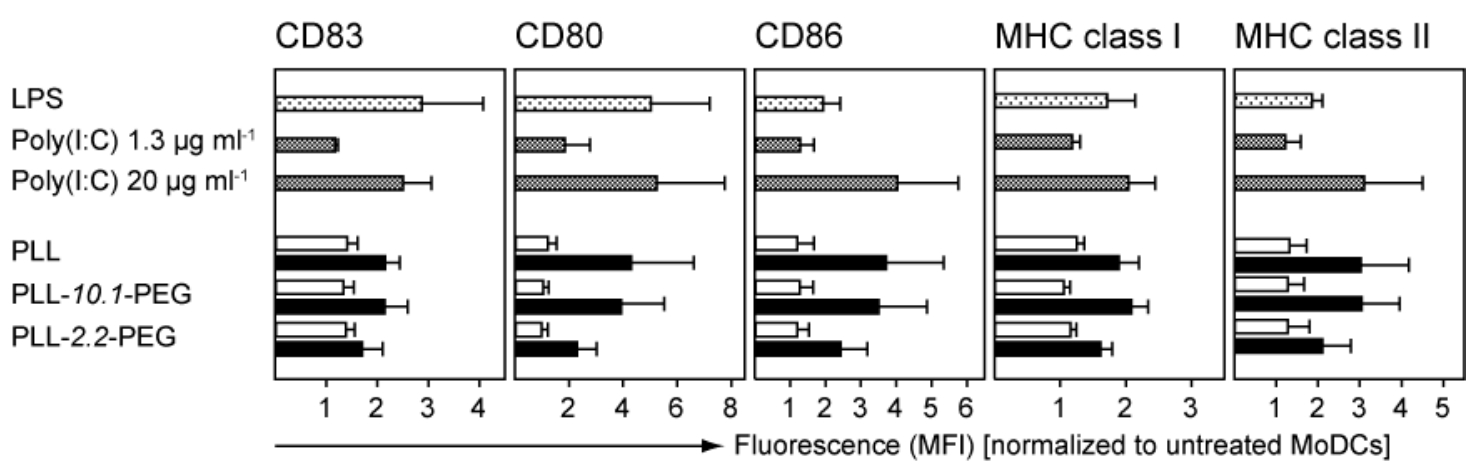

Fig. 4. Expression of maturation-related markers by MoDCs stimulated with free poly $(\mathrm{l}: \mathrm{C})$ or surface-assembled poly(l:C) on PLGA(tt) microspheres. Flow cytometric analyses of the surface expression of CD83, CD80, CD86, class I and class II MHC molecules by MoDCs three days after exposure to $1.3 \mathrm{\mu g} \mathrm{ml}^{-1}$ or $20 \mathrm{\mu g} \mathrm{ml}^{-1}$ free poly(l:C) (dark grey bars), PLGA(tt) microspheres coated with either PLL, PLL-10.1-PEG or PLL-2.2-PEG and loaded with surface-assembled poly(I:C) (black bars). Negative controls were PLGA(tt) microspheres coated with either PLL, PLL-10.1-PEG or PLL-2.2-PEG but without poly(I:C) (open bars). MoDCs treated with LPS were a positive control for the maturation of MoDCs. Mean MFI \pm SD $(n=5)$.

\subsection{Cytokine secretion pattern of MoDCs stimulated with surface-assembled poly(l:C)}


In parallel to the expression of maturation-related surface markers we assessed the secretion of selected cytokines in the supernatant. In particular, we measured the secretion of the inflammatory cytokines IL-6, TNF- $\alpha$ and IL-12p70, as well as the regulatory cytokine IL-10. Results derived from three donors are depicted in Fig. 5.

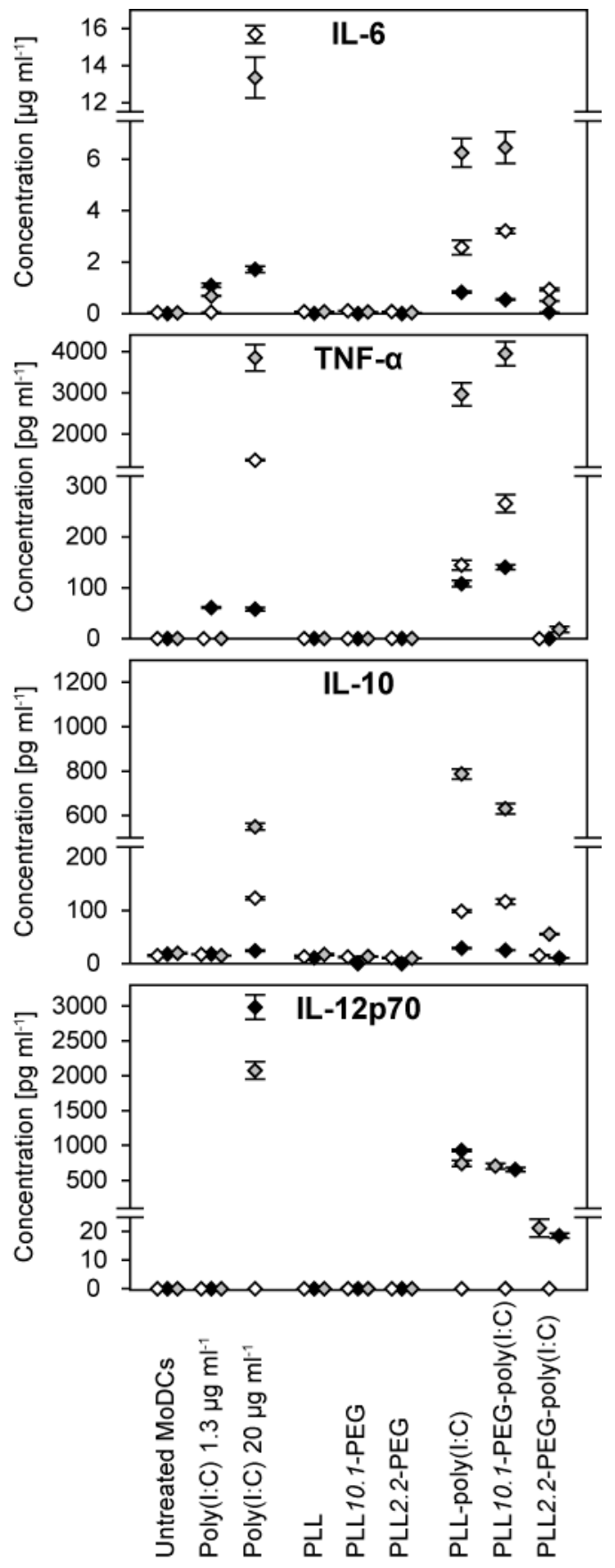

Fig. 5. Cytokine secretion patterns of MoDCs stimulated with either free or surfaceassembled poly $(\mathrm{I}: \mathrm{C})$. MoDCs were treated with surface-assembled poly $(\mathrm{I}: \mathrm{C})$ on either PLL , PLL-10.1-PEG- or PLL-2.2-PEG-coated PLGA(tt) microspheres. For comparison, MoDCs were treated with the same dosage of free poly $(\mathrm{I}: C)$ that was used for surface assembly $\left(1.3 \mathrm{\mu g} \mathrm{ml}^{-1}\right)$. Untreated MoDCs and MoDCs treated with coated PLGA(tt) microspheres without poly $(\mathrm{I}: \mathrm{C})$ were used as background controls. As reference for full maturation MoDCs were treated with $20 \mu \mathrm{g}$ $\mathrm{ml}^{-1}$ free poly(I:C). Supernatants were collected $72 \mathrm{~h}$ after the treatment and the concentrations of IL 6 , TNF $\alpha$, IL 10 and IL 12p70 were analyzed. MoDCs were generated from three different donors (black, gray and open symbols). Mean $\pm \mathrm{SD}, \mathrm{n}=3$.

In response to surface-assembled poly(l:C) MoDCs secreted high levels of all four cytokines. Similar to the expression of the maturation-related surface markers, cytokine secretion depended on the grafting ratio of the coating polymer. PLGA(tt) microspheres coated with 
PLL-2.2-PEG only led to minor cytokine levels. As expected, formulations without poly(I:C) did not induce appreciable cytokine levels but remained on the levels of untreated MoDCs. Hence, the encapsulated tt lacked appreciable effects on cytokine secretion. Free poly(l:C) gave rise to cytokine secretion in a concentration-dependent manner, as demonstrated by high levels for $20 \mathrm{\mu g} \mathrm{ml}^{-1}$ and much lower levels for $1.3 \mu \mathrm{g} \mathrm{ml}^{-1}$. Untreated MoDCs secreted only negligible levels of IL- 6 and IL-10 and no TNF- $\alpha$ or IL-12p70.

It is noteworthy that surface-assembled poly $(\mathrm{I}: \mathrm{C})$ led to higher levels of all four cytokines than the comparable dose of free poly(l:C), $1.3 \mu \mathrm{g} \mathrm{ml}^{-1}$. In contrast, the poly(l:C) concentration of $20 \mathrm{~g} \mathrm{ml}^{-1}$ induced higher IL- 6 and IL-12p70 secretion than surfaceassembled poly(I:C) whereas IL-10 and TNF- $\alpha$ secretions were comparable. Corresponding to the upregulation of the maturation markers, the cytokine secretion levels were highly donor-dependent and varied between the three donors, but without compromising the general trend.

\subsection{Directed migration of MoDCs after stimulation with surface-assembled poly(l:C)}

After pathogen encounter and subsequent maturation of immature DCs, it is crucial that DCs migrate to proximal lymphoid organs for antigen presentation. We assessed the potential of surface-assembled poly $(\mathrm{I}: \mathrm{C})$ to trigger the upregulation of CCR7 in MoDCs as well as the capacity of such stimulated MoDCs to migrate along a CCL21 gradient (Fig. 6). For direct comparison we stimulated MoDC with free poly $(\mathrm{I}: \mathrm{C})$ in a dose equivalent to that used for surface assembly, $1.3 \mathrm{\mu g} \mathrm{ml}^{-1}$. MoDCs were further treated with $20 \mu \mathrm{g} \mathrm{ml}^{-1}$ free poly(l:C) and LPS as references for a distinctly mature phenotype. CCR7 surface-expression was assessed by flow cytometry $72 \mathrm{~h}$ after exposure. PLL-, PLL-10.1-PEG- and PLL-2.2-PEGcoated PLGA(tt) microspheres without poly $(\mathrm{I}: \mathrm{C})$ and immature MoDCs were used as background references. MoDCs were generated from three different donors. The results are presented in Fig. 6.

MoDC exposure to surface-assembled poly(I:C) on PLL- and PLL-10.1-PEG-coated microspheres induced a CCR7 expression which was close to MoDCs matured with a fifteen times higher dosage of free poly(l:C) $\left(20 \mathrm{\mu g} \mathrm{ml}^{-1}\right)$ (Fig.6A). The effect of PLL-2.2-PEGpoly $(\mathrm{I}: \mathrm{C})$ microspheres dropped somewhat. Nevertheless, CCR7 expression levels were still higher than with the equivalent dosage of free poly $(\mathrm{I}: \mathrm{C})$. All microsphere formulations without poly(I:C) as well as MoDCs matured with LPS led to lower CCR7 expression levels. 

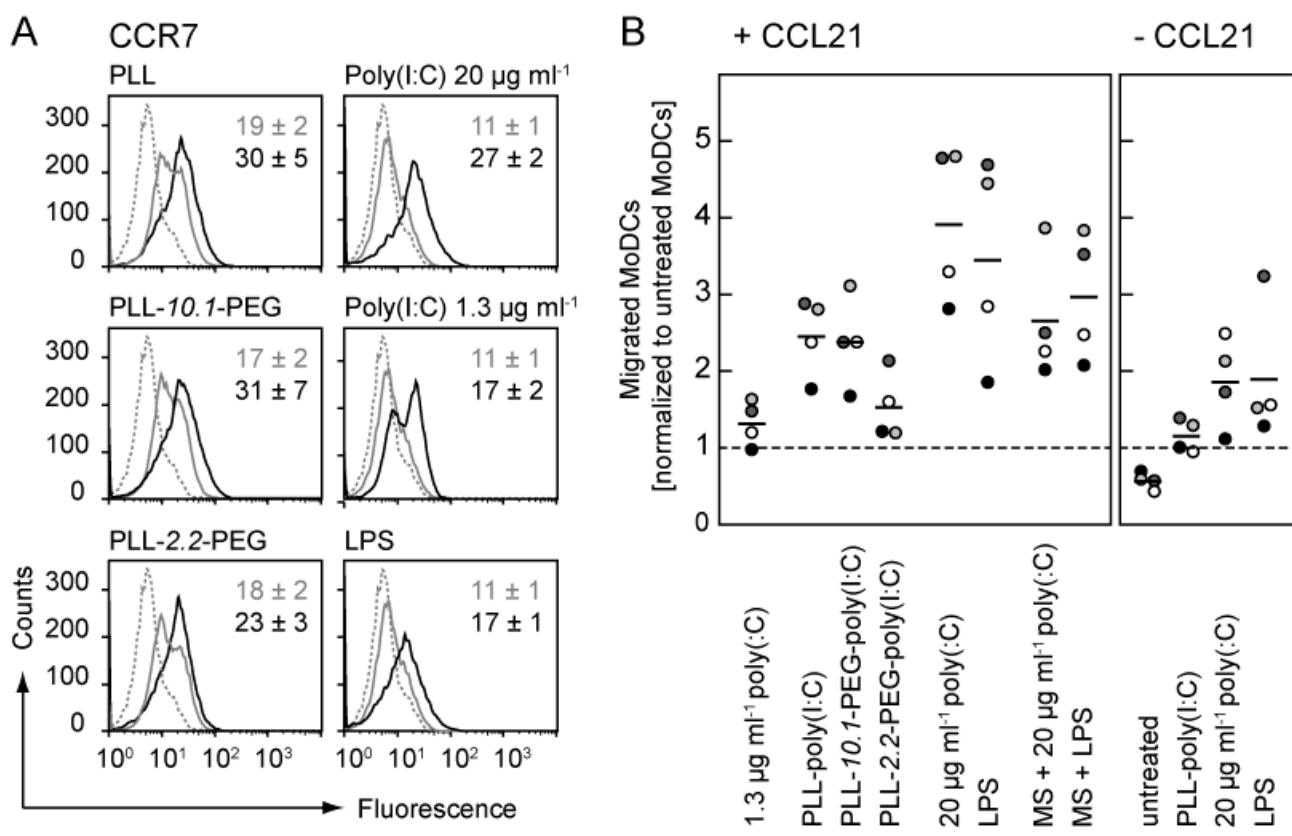

Fig. 6. CCR7 expression and migration capacity of MoDCs stimulated with surfaceassembled poly(I:C). A. Flow cytometric analysis of CCR7 expression on MoDCs. Histogram plots of one representative experiment out of three are shown. Left panels: Black lines: MoDCs upon exposure to poly(I:C) surface-assembled on either PLL-, PLL-10.1-PEGor PLL-2.2-PEG-coated PLGA(tt) microspheres. Grey lines: MoDCs treated with PLL-, PLL10.1-PEG- or PLL-2.2-PEG-coated microspheres without poly(I:C). Dotted lines: isotype controls. Right panels: Black lines: MoDCs treated with either free poly(l:C) $\left(20 \mu \mathrm{g} \mathrm{ml}^{-1}, 1.3\right.$ $\mu \mathrm{g} \mathrm{ml}^{-1}$ ) or LPS. Grey lines: Untreated MoDC were used as background controls. Dotted lines: isotype controls. Numbers indicate the average MFI values of three experiments. Mean MFI $\pm S D, n=3$. B. Chemotaxis Transwell assay to assess CCL21-directed migration of MoDCs in response to surface-assembled poly(l:C) (left panel). MoDCs were treated with surface-assembled poly $(\mathrm{I}: \mathrm{C})$ on three differently coated PLGA(tt) microspheres: PLLpoly(I:C), PLL-10.1-PEG-poly(I:C) and PLL-2.2-PEG-poly(I:C). Positive controls for the full migration potential of mature MoDCs were MoDCs stimulated with either free poly(l:C) or LPS. To identify a potential obstruction of migration due to phagocytosed microspheres MoDCs were incubated with PLL-coated PLGA(tt) microspheres (+MS) for $4 \mathrm{~h}$ and subsequently matured with either free poly(l:C) or LPS equal to the positive controls. Untreated MoDCs were used as reference for normalization. To assess undirected migration the chemotaxis assay was performed without CCL21 (right panel). MoDCs were generated from four different donors (black, dark grey, light grey and open symbols). Dashes indicate means. Each experiment was done in duplicate. 
We further performed a chemotaxis Transwell assay to assess the potential of such treated MoDCs for directed migration towards a CCL21 gradient (Fig. 6B, left panel). As positive controls for the full migration potential of mature MoDCs, MoDCs were treated with free poly(I:C) or LPS. In order to identify an expected obstruction of migration due to phagocytosed microspheres, MoDCs were incubated with PLL-coated PLGA(tt) microspheres for $4 \mathrm{~h}$ and subsequently matured with either free poly(I:C) $\left(20 \mu \mathrm{g} \mathrm{ml}^{-1}\right)$ or LPS as the positive controls. Should an obstruction of migration result from prior phagocytosed microspheres, a reduced number of migrated MoDCs was expected as compared to the positive control of MoDCs treated with free poly(I:C) or LPS alone. To account for undirected migration the assay was also performed without CCL21 (Fig. 6B, right panel). MoDCs were generated from four different donors and the numbers of migrated cells were normalized to the respective reference of untreated, hence immature MoDCs.

MoDCs bearing PLL- and PLL-10.1-PEG-coated microspheres with surface-assembled poly $(\mathrm{I}: \mathrm{C})$ readily migrated along the CCL21 gradient to the bottom chamber of the Transwell unit. Migration of MoDCs fed with PLL-2.2-PEG-poly(I:C) microspheres was less pronounced, consistent with the reduced CCR7 expression (Fig. 6A). Interestingly, the migration rates of MoDCs bearing no microspheres but matured with either $20 \mathrm{\mu g} \mathrm{ml}^{-1}$ free poly (I:C) or LPS were even higher. However, the difference largely dissolved if MoDCs were fed with PLL-coated PLGA(tt) microspheres prior to their maturation with free poly(l:C) or LPS. Overall, the data suggests no major difference between the migration potential of MoDCs stimulated with free or surface-assembled poly $(\mathrm{l}: \mathrm{C})$. Undirected migration through the Transwell unit was lower than migration along the CCL21 gradient. It was the highest with microsphere-free treatments. There was marked variability between donors.

An obstructed migration of MoDCs through the Transwell unit due to phagocytosed microspheres was confirmed (for data see Supplement Fig. 1). Only MoDCs bearing a limited amount of microspheres were able to migrate through the rigid $5 \mu \mathrm{m}$ pores of the membrane whereas MoDCs bearing extra microspheres were retained in the upper chamber of the Transwell unit.

\subsection{PLGA(tt) microsphere phagocytosis by HFFs}

Because of the capacity of fibroblasts as non-professional phagocytes, we evaluated whether PLL- or PLL-g-PEG-coated biodegradable PLGA(tt) microspheres loaded with surface-assembled poly $(\mathrm{I}: \mathrm{C})$ were subject to phagocytosis by dermal fibroblasts and whether phagocytosis was affected by the nature of the PEG corona or the surface assembly of poly(I:C). 
A

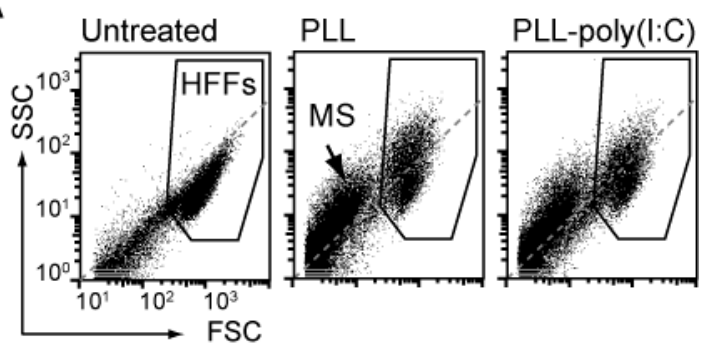

B

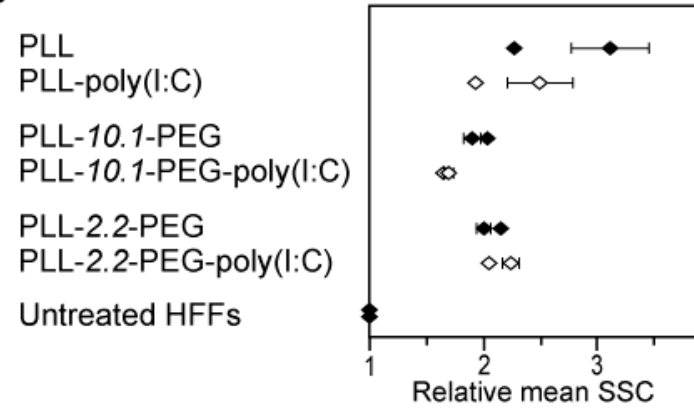

C

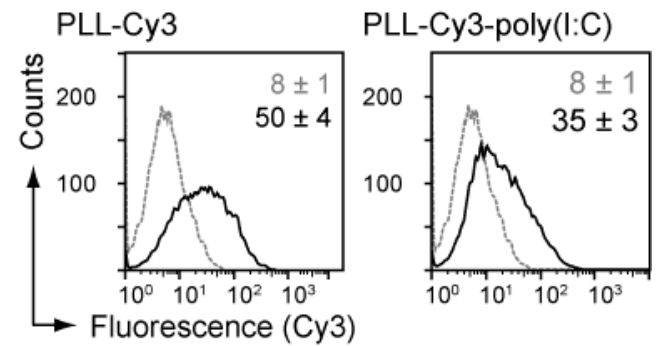

D

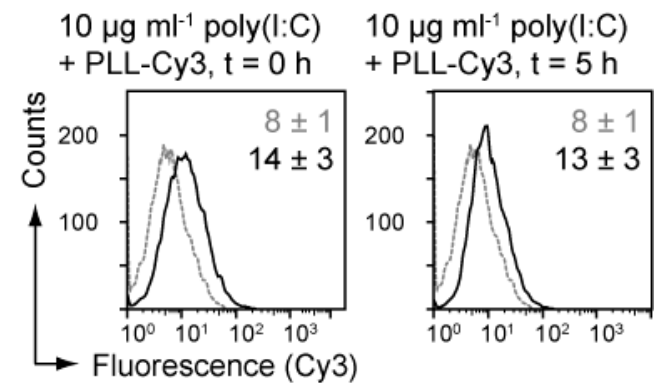

Fig. 7. Flow cytometric analysis of the phagocytosis of PLGA(tt) microspheres by HFFs. A. FFC/SSC dot plots of untreated HFFs (left panel) and HFFs after phagocytosis of PLL-coated PLGA(tt) microspheres without (middle panel) and with surface-assembled poly(I:C) (right panel). Morphological properties such as cell size (FSC) and cell granularity (SSC) were used for gating. The gate encloses the HFF population. MS: free microspheres. The shift of the HFF population towards an increased SSC signal visualizes the effect of phagocytosed microspheres on HFF granularity. B. SSC signals of HFFs after incubation with various microsphere formulations, normalized by SSC signals of untreated HFFs. Formulations without poly(I:C): PLL-, PLL-10.1-PEG- and PLL-2.2PEG-coated microspheres (black diamonds). Formulations loaded with surface assembled poly(I:C): PLL-poly(I:C), PLL-10.1-PEGpoly(I:C) and PLL-2.2-PEG-poly(I:C) (open diamonds). Untreated HFFs were used as reference. Two independent experiments with different donors: (i) mean \pm SD of triplicates ( $n=3$ ), and (ii) $n=1$, respectively. C-D. Inhibitory effect of free and surfaceassembled poly(I:C) on the phagocytic activity of HFFs. Histogram plots illustrating the distribution of HFFs containing phagocytosed fluorescent microspheres. Numbers indicate MFI values of untreated HFFs as reference (grey) and HFFs after the respective treatment with fluorescent microspheres (black). Numbers indicate means \pm SD of triplicates $(n=3)$ within experiment. C. HFFs treated with PLL-Cy3coated microspheres without poly(I:C) (PLLCy3) as positive control for phagocytosis (left panel). HFFs treated with surface-assembled poly(I:C) on PLL-Cy3-coated PLGA(tt) microspheres, PLL-Cy3-poly(I:C) (right panel) $D$. HFFs treated with fluorescent PLL-Cy3coated PLGA(tt) microspheres concurrently (t $=0 \mathrm{~h})$ and $5 \mathrm{~h}$ after $(\mathrm{t}=5 \mathrm{~h})$ the stimulation with $10 \mu \mathrm{g} \mathrm{ml}^{-1}$ free poly(l:C). 
Primary human foreskin fibroblasts (HFFs) were incubated for $48 \mathrm{~h}$ with three differently coated PLGA(tt) microsphere formulations, either without or with surface-assembled poly(I:C). Coatings were made with PLL, PLL-10.1-PEG or PLL-2.2-PEG. Untreated HFFs were used as reference. Phagocytosis by HFFs was qualitatively assessed using the mean SSC signal to visualize the granularity of the HFFs. SSC was previously established as a qualitative parameter for phagocytosis by MoDCs and HFFs using PS microspheres (Wattendorf et al., 2006; Hafner et al., 2012).

The FSC/SSC dot plots illustrate the gated HFF-population as well as the added microspheres (MS) (Fig. 7A). A pronounced shift towards 2- to 3-fold higher mean SCC values was observed after HFF incubation with either PLL-coated PLGA(tt) microspheres with or without surface assembled poly(I:C). In general, HFF exposure to PLGA(tt) microspheres, regardless whether decorated with a PEG corona or with surface-assembled poly $(I: C)$, led to increased mean SSC signals as compared to untreated HFFs, thus indicating microsphere phagocytosis (Fig. 7B). For PLL- and PLL-10.1-PEG-coated microspheres surface-assembled poly(I:C) on PLL-coated PLGA(tt) microspheres showed a slight trend towards reduced phagocytosis, but not for PLL-2.2-PEG-coated microspheres. In order to further investigate a potential influence of poly $(\mathrm{I}: \mathrm{C})$ on the phagocytic activity of HFFs we also used fluorescent PLL-Cy3-coated PLGA(tt) microspheres, without and with surface-assembled poly(I:C). Further, HFFs were treated with $10 \mu \mathrm{g} \mathrm{ml}^{-1}$ free poly(I:C) either $5 \mathrm{~h}$ prior to or concurrent with the administration of fluorescent PLL-Cy3-coated PLGA(tt) microspheres. As positive control for phagocytosis otherwise untreated HFFs were exposed to fluorescent PLL-Cy3-coated microspheres, while untreated HFFs were used as background control. In order to compare free versus surface assembled poly(I:C), HFFs were incubated with surface-assembled poly(I:C) on PLL-Cy3-coated PLGA(tt) microspheres, denoted as PLL-Cy3-poly(l:C). Phagocytosis of fluorescent microspheres was analyzed by flow cytometry using the Cy3-fluorescence intensity. Results are presented in Fig. 7C-D.

PLL-Cy3-coated PLGA(tt) microspheres were readily phagocytosed by otherwise unstimulated HFFs (Fig. 7C, left panel). This was indicated by an increased MFI of the Cy3 signal. Surface-assembled poly(I:C) on PLL-Cy3-coated PLGA(tt) microspheres resulted in a markedly reduced MFI of the Cy3 signal (Fig. 7C, right panel). Finally, regardless of the time point of poly $(\mathrm{I}: \mathrm{C})$ treatment, HFFs stimulated with high doses of free poly $(\mathrm{I}: \mathrm{C})\left(10 \mu \mathrm{g} \mathrm{ml}^{-1}\right)$ exhibited distinctly lower MFIs of the Cy3 signal close to untreated HFFs (Fig. 7D). This indicates that high dosages of free poly $(\mathrm{I}: \mathrm{C})$ instantaneously inhibited the phagocytosis of PLGA(tt) microspheres by HFFs. 


\subsection{Class I and class II MHC molecule surface expression by HFFs after exposure to} surface-assembled poly(l:C)

In contrast to MoDCs, fibroblasts express TLR3 on their surface, endowing them to detect dsRNA in the extracellular space. The stimulation of HFFs with viruses, viral dsRNA or poly $(\mathrm{I}: \mathrm{C})$ has been repeatedly reported to upregulate the expression of class I MHC molecules (Steinmassl et al., 1994). Moreover, poly(I:C) was found to upregulate the expression of class II MHC molecules when transduced into the cytoplasm of several types of non-hematopoietic cells (Suzuki et al., 1999). In order to avoid the danger of an overstimulation of the immune system it may thus be beneficial to restrict the dose of poly $(\mathrm{I}: \mathrm{C})$ to the minimum possible and control its contact with non-hematopoietic bystander cells. In this context we studied the stimulation of HFFs with surface-assembled poly $(\mathrm{I}: \mathrm{C})$ on three PLGA(tt) microsphere formulations. HFFs were treated with surface-assembled poly(I:C) on PLL-, PLL-10.1-PEG- and PLL-2.2-PEG-coated PLGA(tt) microspheres. As controls HFFs were also treated with free poly(I:C) at $0.1 \mu \mathrm{g} \mathrm{ml}^{-1}, 1 \mu \mathrm{g} \mathrm{ml}^{-1}$ and $10 \mathrm{gg} \mathrm{ml}^{-1}$. Negative controls were coated PLGA(tt) microspheres without poly $(\mathrm{l}: \mathrm{C})$ and untreated HFFs. Class I and class II MHC molecule expression was assessed by flow cytometry (Fig. 8).

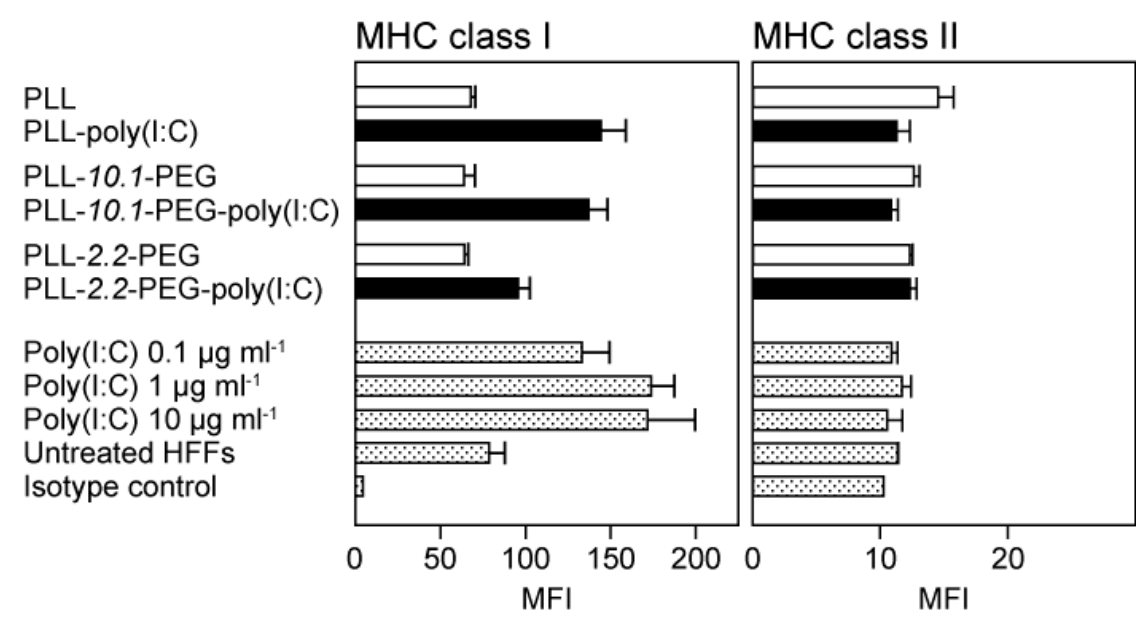

Fig. 8. Flow cytometric analysis of class I (left panel) and class II (right panel) MHC molecule expression by HFFs after incubation with various PLGA(tt) microsphere formulations either loaded with surface-assembled poly(I:C) (black bars) or without poly $(\mathrm{I}: \mathrm{C})$ (open bars). Formulations without poly(I:C) were PLL-, PLL-10.1-PEG- and PLL-2.2-PEGcoated microspheres. Formulations loaded with surface assembled poly $(\mathrm{I}: \mathrm{C})$ were PLLpoly(I:C), PLL-10.1-PEG-poly(I:C) and PLL-2.2-PEG-poly(I:C). Controls were: HFFs treated with $0.1 \mathrm{\mu g} \mathrm{ml}^{-1}, 1 \mu \mathrm{g} \mathrm{ml}^{-1}$ and $10 \mu \mathrm{g} \mathrm{ml}^{-1}$ free poly(I:C) to compare the effects of free versus surface-assembled poly $(\mathrm{I}: \mathrm{C})$, untreated HFFs and an IgG isotype control (dotted bars). Mean MFI \pm SD of triplicates $(n=3)$ within experiment. 
Throughout, microspheres loaded with surface-assembled poly $(\mathrm{l}: \mathrm{C})$ gave rise to class I MHC molecule upregulation. We found similar class I MHC molecule expression levels in all poly(I:C)-formulations except for PLL-2.2-PEG showing somewhat lower levels. Comparable levels were found with $0.1 \mathrm{\mu g} \mathrm{ml}^{-1}$ free poly $(\mathrm{I}: C)$. All samples and controls without poly(I:C) showed class I MHC molecule expressions comparable to untreated HFFs. Neither the formulations with surface-assembled nor free poly $(\mathrm{I}: \mathrm{C})$ induced class II MHC molecule upregulation on HFFs. 


\section{Discussion}

In two previous studies with poly(I:C) we used negatively charged carboxylated PS microspheres of $5 \mu \mathrm{m}$ diameter with a narrow size distribution, uniform shape and a low propensity towards agglomeration (Hafner et al., 2011; Hafner et al., 2012). Here we expanded our concept to biodegradable PLGA(tt) microspheres, combining a clinically relevant biomaterial with a previously well documented model antigen (Men et al., 1995; Men et al., 1999; Waeckerle-Men et al., 2006).

We modified the surface properties of the PLGA(tt) microspheres, such as electrical charge and PEGylation degree, using PLL- or PLL-g-PEG coatings with variable PEG densities, and by the subsequent surface assembly of poly(I:C). The immunostimulatory potential of such poly(I:C)-loaded PLGA(tt) microspheres was evaluated on MoDCs as well as HFFs, chosen as a model for non-hemopoietic bystander cells. As to the MoDC part, the focus was on (i) microsphere phagocytosis, (ii) expression of maturation-related surface markers, (iii) cytokine secretion pattern and (iv) migration capacity after phagocytosis of PLGA(tt) microspheres loaded with surface-assembled poly $(\mathrm{I}: \mathrm{C})$. With respect to HFF we studied the previously described inhibitory effect of poly $(\mathrm{l}: \mathrm{C})$ on the $(\mathrm{v})$ phagocytosis of the microspheres as well as the effect on the (vi) expression of class I and class II MHC molecules (Hafner et al., 2012). Overall, we found both equal outcomes as well as distinct differences between the PLGA(tt) system and the previously investigated PS model (Hafner et al., 2011; Hafner et al., 2012) as discussed in the following sections.

\subsection{Immunostimulatory efficacy of surface-assembled poly(I:C) on PLGA(tt) microspheres}

Increases in the immunostimulatory efficacy of poly $(\mathrm{I}: \mathrm{C})$ when surface-assembled on microspheres as compared to free poly(I:C) are documented (Wischke et al., 2009; Hafner et al., 2011). Wischke et al. (2009) demonstrated that poly(I:C), when surface-assembled on diethylaminoethyl dextran-coated PLGA microspheres, stimulated the maturation of MoDCs with an increased efficacy of almost one order of magnitude as compared to free poly(I:C). The authors proposed that when bound to the surface of microspheres poly $(I: C)$ may interact more efficiently with the TLR3 receptor than free ligand (Wischke et al., 2009). Using a PS microsphere model system, we proposed that this increase is due to a synergy between TLR3 and phagocytic signaling. By exploring dose-efficacy relationships in MoDCs we found multiple phagocytosis events to be key to enhance efficacy (Hafner et al., 2011). 
In the present work with PLGA microspheres we confirmed a similar outcome for PLL- and PLL-g-PEG-coated PLGA(tt) microspheres with surface-assembled poly(l:C). Boosted immunostimulatory efficacy was manifest by largely enhanced upregulation of maturationassociated surface-markers of MoDCs (Fig. 3-4) as well as increased cytokine secretion (Fig. 5), CCR7 expression and enhanced MoDC migration as compared to the same dose of free poly(l:C) (Fig. 6).

Nevertheless, given the fact that PLL-2.2-PEG coated microspheres were readily phagocytosed by MoDCs, it was striking that the immunostimulatory efficacy of surfaceassembled poly(l:C) on PLL-2.2-PEG-coated PLGA(tt) microspheres was below that on PLLor PLL-10.1-PEG-coated microspheres. Hypothetically, we considered that the amount of surface-assembled poly(I:C) on PLL-2.2-PEG-coated PLGA(tt) microspheres was not sufficient to efficiently trigger MoDC maturation. This hypothesis complies with the findings of Wischke et al. (2009) who observed a dose dependency in the efficacy of surfaceassembled poly $(\mathrm{I}: \mathrm{C})$ on PLGA microspheres. Even so, the result remains in contrast with our previous data obtained with PS microspheres. Based on the same experimental protocoll, we then found that even lower dosages of surface-assembled poly(I:C) did not compromise the upregulation of maturation-related surface markers on MoDCs (Hafner et al., 2011).

We tend to attribute this discrepancy to contrasts between PLGA(tt)- and PS microspheres. For instance, the biodegradability of PLGA(tt)- versus the non-biodegradability of PS microspheres may play a critical role, as well as the wider particle size distribution of the PLGA(tt) microspheres versus the uniform particle size of $5 \mu \mathrm{m}$ of the PS microspheres, respectively. Indeed, particle size was found a key aspect for the synergistic effect of endocytosis and TLR signaling (Sharp et al., 2009). Phagocytosis of microspheres and concurrent exposure of poly $(\mathrm{I}: \mathrm{C})$ or other TLR agonists enhanced the secretion of IL-1 $\beta$ by murine bone marrow-derived DCs as compared to TLR agonists alone. Interestingly, the enhanced IL-1 $\beta$ secretion was particle size dependent. Out of $0.43 \mu \mathrm{m}, 1 \mu \mathrm{m}, 10 \mu \mathrm{m}$ and $32 \mu \mathrm{m}$ samples of PS microspheres, the $1 \mu \mathrm{m}$ microspheres performed best, followed by a markedly reduced performance of the $0.43 \mu \mathrm{m}$ sample, while PS microspheres of $10 \mu \mathrm{m}$ and $32 \mu \mathrm{m}$ in diameter only weakly enhanced IL-1 $\beta$ secretion, due to limited or lacking phagocytosis (Sharp et al., 2009).

Considering that the PLGA(tt) microspheres used in the present study exhibited a wide particle size distribution, we may expect various endocytic mechanisms to be involved in parallel. Amongst other parameters, endocytic mechanisms are size specific: Ingestion of particles larger than $0.5 \mu \mathrm{m}$ occurs via phagocytosis, while smaller particles are internalized by receptor-mediated clathrin-dependent or clathrin-independent pathways, as previously reviewed (Kumari et al., 2010). While endocytosis of the rather narrow-sized PS microsphere 
population in our previous study may be restricted to a single mechanism (Hafner et al., 2011), the uptake of the wide-sized PLGA(tt) microsphere population may follow more than one mechanism.

\subsection{Chemotactic migration of $M o D C$ in response to surface-assembled poly(l:C)}

With a chemotaxis Transwell assay we successfully demonstrated a CCL21-directed migration of CCR7 positive MoDCs matured with surface-assembled poly(l:C). Although comparable levels of CCR7 were expressed by MoDCs stimulated with free and surfaceassembled poly $(\mathrm{I}: \mathrm{C})$, less MoDCs migrated in response to surface-assembled poly(l:C). Interestingly, the retention of MoDCs in the upper chamber of the Transwell unit correlated with a high internal granularity and a strong Cy3-fluorescence, hence with the amount of phagocytosed microspheres (see Supplement Fig. 1). This indicated that phagocytosis of a critical number or volume of PLGA (tt) microspheres made the MoDCs bulkier and resulted in a steric obstruction of the migration across the pores of the Transwells in vitro. Obstruction may be lessened by reducing the microsphere load per cell. Indeed, previous studies with a similar assay but with a $40 \%$ reduced PLGA microsphere supply per cell, showed no appreciable obstruction of migration (Waeckerle-Men et al., 2004; Fischer et al. 2007).

Nevertheless - in order to put this into perspective - the observed in vitro obstruction is unlikely to happen under in vivo conditions, where DCs are known to be flexible enough to squeeze themselves through connective tissue and across epithelial junctions. So, in the first place, the observed obstruction of microsphere-loaded MoDCs, when migrating across the rigid $5 \mu \mathrm{m}$ pores of the Transwell membrane, is primarily a physical effect of the experimental set-up, and not due to a lack in CCR7 expression.

\subsection{PEGylation and microsphere phagocytosis by professional and non-professional phagocytes}

Previously we described that the phagocytosis of PLL-g-PEG-coated microspheres was strongly diminished by a sufficiently dense PEG corona. For instance, phagocytosis of PLLg-PEG-coated PS microspheres (Wattendorf et al., 2006; Hafner et al., 2011) or hollow polyelectrolyte microcapsules (Wattendorf et al., 2008) by professional phagocytes, i.e. MoDCs and macrophages, was efficiently reduced at grafting ratios $g$ of 5.7 or smaller. To inhibit the phagocytosis of PS microspheres by non-professional phagocytes like HFFs, even a grafting ratio of 10.1 was sufficient (Hafner et al., 2012). 
In contrast, in the present study all PLGA(tt) microsphere formulations were readily phagocytosed by MoDCs (Fig. 2) as well as HFFs (Fig. 7), regardless of the polymer coating. This leads us to conclude that PLL-g-PEG-coated PLGA(tt) microspheres are more susceptible to phagocytosis than equally coated PS microspheres or hollow polyelectrolyte microcapsules. The discrepancy indicates a less homogenous or less dense PEG corona on PLGA(tt) microspheres when coated with PLL-g-PEG polymers, possibly a result of a less efficient surface assembly of the PLL backbone on PLGA surfaces. The less negative $\zeta-$ potential of PLGA(tt) microspheres $(7 \pm 8 \mathrm{mV})$ as compared to uncoated PS microspheres ($36 \pm 4 \mathrm{mV}$ ) (Fig. 1) (Hafner et al., 2011) as well as the only moderately positive $\zeta$-potentials of coated PLGA(tt) microspheres clearly support this assumption. A more specific hypothesis to explain less homogenous or less dense PEG coronas on PLGA(tt) microspheres could be an inhomogeneous charge distribution on the surface, presumably resulting from the uneven, granular surface caused by phase-separated domains of poly(L-lactic acid) and poly(L-glycolic acid) on the surface of PLGA microspheres after solvent extraction, which we previously observed by atomic force microscopy (Csaba et al., unpublished data from our lab). Also an influence of the PVA typically used as dispersion agent during microsphere preparation could have an impact on the coating process. In conclusion, the stealth quality of the PEG corona on PLGA microspheres may need technical improvement. Nonetheless, even though the PEG coatings on PLGA(tt) microspheres turned out to be less complete than as previously observed on PS microspheres (Hafner et al., 2011), the subsequent surface assembly of poly(I:C) was equally efficient as confirmed by highly negative $\zeta$ potentials (Fig. 1).

\subsection{Poly(I:C)-mediated MHC molecule upregulation on HFFs}

Interestingly, all PLGA(tt) microsphere formulations with surface-assembled poly(l:C) readily induced upregulation of class I MHC molecule expression by HFFs. This was not unexpected because free poly $(\mathrm{I}: \mathrm{C})$ concentrations as low as $0.1 \mathrm{\mu g} \mathrm{ml}^{-1}$ induced comparable class I MHC molecule upregulation (Fig. 8).

In addition to an enhancement of class I MHC expression, poly $(\mathrm{I}: \mathrm{C})$ was previously claimed to trigger the expression of class II MHC molecules when delivered into the cytoplasm of non-immune cells. In fact, Suzuki et al. (1999) reported on an aberrant class II MHC molecule expression in an array of non-immune cells such as, e.g., thyroid cells, COS cells and fibroblasts, after translocation of poly(I:C), when using the liposomal transfection reagent lipofectamine. The significance of this result lies in the observation that class II MHC molecule expression by non-immune cells was associated with the development of 
autoimmune diseases as previously reviewed (Hafner et al., 2013). Notably, in our previous study with PS microspheres both free and surface-assembled poly(I:C) failed to affect class II MHC molecule expression by HFFs and HeLa cells (Hafner et al., 2012).

In accordance with this data, surface-assembled poly(I:C) on PLGA(tt) microspheres did not induce class II MHC molecule expression by HFFs (Fig. 8 right panel). According to Suzuki et al. (1999), the upregulation of class II MHC specifically requires the translocation of poly(I:C) into the cytoplasm, which does not occur with PLGA microspheres (Thiele et al., 2003). This argues in favor of the safety of microparticulate PLGA formulations for poly(I:C) delivery.

\subsection{Impact of free and surface-assembled poly(l:C) on microsphere phagocytosis}

Neither on PLGA(tt) (Fig. 2) nor on PS microspheres (Hafner et al., 2011) could surfaceassembled poly $(\mathrm{I}: \mathrm{C})$ influence their phagocytosis by MoDCs. In contrast, free as well as surface-assembled poly $(\mathrm{I}: \mathrm{C})$ were previously found to strongly inhibit PS microsphere phagocytosis of HFFs in a dose-dependent fashion (Hafner et al., 2012). In the present work all PLGA(tt) microsphere formulations, regardless of whether decorated with a PEG corona or bearing surface-assembled poly $(\mathrm{I}: \mathrm{C})$, were readily phagocytosed, as indicated by increased mean SSC signals as compared to untreated HFFs (Fig. 7B) as standard. As discussed above, this discrepancy could also be attributed to lower doses of surfaceassembled poly $(\mathrm{I}: \mathrm{C})$, because the inhibiting effect of poly $(\mathrm{I}: \mathrm{C})$ on phagocytosis was found to be dose-sensitive (Hafner et al., 2012). Unlike the previously investigated PS microspheres (Hafner et al., 2012), the SSC signals from HFFs bearing phagocytosed PLGA(tt) microspheres were not sensitive enough for more than a rough estimation of phagocytosis (Fig. 7B). As an explanation we point to the high fraction of small particle sizes typical for the PLGA(tt) microspheres, as demonstrated by their rather wide volume size distribution $(D(v, 0.1)=1.7 \mu \mathrm{m} ; D(v, 0.9)=7.1 \mu \mathrm{m})$. In contrast, the PS microspheres had a uniform particle size of $5 \mu \mathrm{m}$ (Hafner et al., 2012). Hence, the granularity of HFFs resulting from phagocytosed PLGA(tt) microspheres is expected to be minor than that after phagocytosis of PS microspheres. Accordingly, the SSC signal-based trends towards reduced phagocytosis by PLL-10.1-PEG coatings and surface-assembled poly(I:C), respectively, were weak. However, by fluorescence analysis of the PLL-Cy3-coated PLGA(tt) microspheres, surfaceassembled poly $(\mathrm{I}: \mathrm{C})$ was clearly found to diminish the phagocytosis of such microspheres by HFFs (Fig. 7C). The relevance of this observation may be stressed by this rationale: Diminished phagocytosis of poly(l:C)-loaded microspheres by bystander cells such as fibroblasts at the site of injection may limit the loss of microspheres to such cells, raise the 
chance for professional APCs to get hold of the microspheres, and thus lower the total dose of microspheres necessary to induce the desired immunostimulatory response.

We also confirmed our previous findings that high dosages of free poly $(\mathrm{I}: \mathrm{C})$ efficiently inhibited the phagocytosis of microspheres by HFFs (Hafner et al., 2012). Indeed, a high dose of free poly $(I: C)$ almost completely stopped the phagocytosis of fluorescent PLL-Cy3coated PLGA(tt) microspheres (Fig. 7D), regardless of the broad particle size distribution of the PLGA(tt) microspheres.

A similar phenomenon has been observed with MoDCs as well. The stimulation of immature MoDCs with TLR3 ligands, although not instantaneously, resulted in a discontinuation of their phagocytotic activity (Weck et al., 2007). This was thought to preserve the set of pathogen-derived antigens for their subsequent presentation to naïve $T$ cells in lymphoid organs. Notably, under specific conditions, fibroblasts were observed to express class II MHC molecules and present foreign antigens to T cells, such as in the environment of lymphoid organs (Kundig et al. 1995) and in vitro after treatment with IFN-Y (Collins et al., 1984). Thus, similar to professional APCs, inhibition of phagocytosis by fibroblasts could help to maintain peripheral tolerance.

A further explanation that deserves consideration is that some viruses use endocytic or phagocytic mechanisms of the cell to gain intracellular access (Mercer et al., 2010). The discontinuation of phagocytosis after an encounter with extracellular dsRNA, a viral danger signal, may thus serve the cell as a measure to shield from viral infection. Nevertheless, at this time, the underlying mechanisms for poly $(\mathrm{I}: \mathrm{C})$ to halt phagocytosis, as observed in HFFs, as well as its biological function need to be subject to further studies.

\subsection{Translational potential}

When considering the translational potential of the investigated PLGA formulations one needs to be aware that a clinically approved poly(I:C)/PLGA formulation is far from being implemented. In spite of the established safety record of PLGA microspheres for parenteral use in humans, its combination with poly(I:C) remains in need of further efforts, ranging from preformulation and formulation studies up to preclinical and clinical investigations. It is still open how formulations as complex as the presented one can be translated from bench to bedside. Investment into process simplification, ease of upscaling and straightforward quality assurance will be essential. Even more so, because of the massive medical and public interest in the safety of vaccines, formulations containing immunostimulatory agents will continue to be under heavy toxicological scrutiny. But therapeutic vaccines against lifethreatening diseases represent an appealing option to go forward. 


\section{Conflict of interest statement}

The authors declare no conflict of interest.

\section{Acknowledgements}

The authors thank Stéphanie Pasche for the synthesis of PLL-g-PEG copolymers and Noemi Csaba as well as Stefan Fischer for helpful instructions for the preparation of PLGA(tt) microspheres. This project was generously funded by the Swiss National Science Foundation SNF (3200B0 102007).

\section{References}

Ammi, R., De Waele, J., Willemen, Y., Van Brussel, I., Schrijvers, D.M., Lion, E., Smits, E.L.J., 2015. Poly(I:C) as cancer vaccine adjuvant: Knocking on the door of medical breakthroughs. Pharmacology \& Therapeutics 146, 120-131.

Audran, R., Men, Y., Johansen, P., Gander, B., Corradin, G., 1998. Enhanced immunogenicity of microencapsulated tetanus toxoid with stabilizing agents. Pharmaceutical Research 15, 1111-1116.

Bottazzo, G.F., Dean, B.M., McNally, J.M., Mackay, E.H., Swift, P.G.F., Gamble, D.R., 1985. In situ characterization of autoimmune phenomena and expression of HLA molecules in the pancreas of diabetic insulitis. New England Journal of Medicine 313, 353-360.

Collins, T., Korman, A.J., Wake, C.T., Boss, J.M., Kappes, D.J., Fiers, W., Ault, K.A., Gimbrone, M.A., Strominger, J.L., Pober, J.S., 1984. Immune interferon activates multiple class-II major histocompatibility complex genes and the associated invariant chain gene in human-endothelial cells and dermal fibroblasts. Proceedings of the National Academy of Sciences of the United States of America-Biological Sciences 81, 4917-4921.

Denis-Mize, K.S., Dupuis, M., MacKichan, M.L., Singh, M., Doe, B., O'Hagan, D., Ulmer, J.B., Donnelly, J.J., McDonald, D.M., Ott, G., 2000. Plasmid DNA adsorbed onto cationic microparticles mediates target gene expression and antigen presentation by dendritic cells. Gene Therapy 7, 2105-2112.

Evans, J.T., Ward, J.R., Kern, J., Johnson, M.E., 2004. A single vaccination with proteinmicrospheres elicits a strong CD8 T-cell-mediated immune response against Mycobacterium tuberculosis antigen Mtb8.4. Vaccine 22, 1964-1972.

Ewel, C.H., Sobel, D.O., Zeligs, B.J., Bellanti, J.A., 1992. Poly-I-C accelerates development of diabetes mellitus in diabetes prone BB rat. Diabetes 41, 1016-1021. 
Fischer, S., Uetz-von Allmen, E., Waeckerle-Men, Y., Groettrup, M., Merkle, H.P., Gander, B., 2007. The preservation of phenotype and functionality of dendritic cells upon phagocytosis of polyelectrolyte-coated PLGA microparticles. Biomaterials 28, 9941004.

Freitas, S., Merkle, H.P., Gander, B., 2005a. Microencapsulation by solvent extraction/evaporation: reviewing the state of the art of microsphere preparation process technology. Journal of Controlled Release 102, 313-332.

Freitas, S., Rudolf, B., Merkle, H.P., Gander, B., 2005b. Flow-through ultrasonic emulsification combined with static micromixing for aseptic production of microspheres by solvent extraction. European Journal of Pharmaceutics and Biopharmaceutics 61, 181-187.

Hafner, A.M., Burschowsky, D., Corthesy, B., Textor, M., Merkle, H.P., 2012. Surface assembly of poly(l:C) on PEGylated microspheres to shield from adverse interactions with fibroblasts. Journal of Controlled Release 159, 204-214.

Hafner, A.M., Corthesy, B., Merkle, H.P., 2013. Particulate formulations for the delivery of poly(I:C) as vaccine adjuvant. Advanced Drug Delivery Reviews 65, 1386-1399.

Hafner, A.M., Corthesy, B., Textor, M., Merkle, H.P., 2011. Tuning the immune response of dendritic cells to surface-assembled poly(I:C) on microspheres through synergistic interactions between phagocytic and TLR3 signaling. Biomaterials 32, 2651-2661.

Hanes, J., Cleland, J.L., Langer, R., 1997. New advances in microsphere-based single-dose vaccines. Advanced Drug Delivery Reviews 28, 97-119.

Jackson, R., McNicol, A.M., Farquharson, M., Foulis, A.K., 1988. Class-II MHC expression in normal adrenal cortex and cortical cells in autoimmune Addisons disease. Journal of Pathology 155, 113-120.

Jilek, S., Merkle, H.P., Walter, E., 2005. DNA-loaded biodegradable microparticles as vaccine delivery systems and their interaction with dendritic cells. Advanced Drug Delivery Reviews 57, 377-390.

Jin, B., Sun, T., Yu, X.-H., Liu, C.-Q., Yang, Y.-X., Lu, P., Fu, S.-F., Quu, H.-B., Yeo, A.E.T., 2010. Immunomodulatory Effects of dsRNA and Its Potential as Vaccine Adjuvant. Journal of Biomedicine and Biotechnology. DOI:10.1155/2010/690438

Johansen, P., Estevez, F., Zurbriggen, R., Merkle, H.P., Gluck, R., Corradin, G., Gander, B., 2000a. Towards clinical testing of a single-administration tetanus vaccine based on PLA/PLGA microspheres. Vaccine 19, 1047-1054.

Johansen, P., Men, Y., Merkle, H.P., Gander, B., 2000b. Revisiting PLA/PLGA microspheres: an analysis of their potential in parenteral vaccination. European Journal of Pharmaceutics and Biopharmaceutics 50, 129-146. 
Kohn, L.D., Shimojo, N., Kohno, Y., Suzuki, K., 2000. An animal model of Graves' disease: understanding the cause of autoimmune hyperthyroidism. Reviews in endocrine \& metabolic disorders 1, 59-67.

Kumari, S., Swetha, M.G., Mayor, S., 2010. Endocytosis unplugged: multiple ways to enter the cell. Cell Research 20, 256-275.

Kundig, T.M., Bachmann, M.F., Dipaolo, C., Simard, J.J.L., Battegay, M., Lother, H., Gessner, A., Kuhlcke, K., Ohashi, P.S., Hengartner, H., Zinkernagel, R.M., 1995. Fibroblasts as efficient antigen-presenting cells in lymphoid organs. Science 268 , 1343-1347.

Lang, K.S., Recher, M., Junt, T., Navarini, A.A., Harris, N.L., Freigang, S., Odermatt, B., Conrad, C., Ittner, L.M., Bauer, S., Luther, S.A., Uematsu, S., Akira, S., Hengartner, H., Zinkernagel, R.M., 2005. Toll-like receptor engagement converts T-cell autoreactivity into overt autoimmune disease (vol 11, pg 138, 2005). Nature Medicine 11, 1256-1256.

Martins, K.A., Bavari, S., Salazar, A.M., 2015. Vaccine adjuvant uses of poly-IC and derivatives. Expert Review of Vaccines 14, 447-459.

Matsumoto, M., Kikkawa, S., Kohase, M., Miyake, K., Seya, T., 2002. Establishment of a monoclonal antibody against human Toll-like receptor 3 that blocks double-stranded RNA-mediated signaling. Biochemical and Biophysical Research Communications 293, 1364-1369.

Men, Y., Audran, R., Thomasin, C., Eberl, G., Demotz, S., Merkle, H.P., Gander, B., Corradin, G., 1999. MHC class I- and class II-restricted processing and presentation of microencapsulated antigens. Vaccine 17, 1047-1056.

Men, Y., Tamber, H., Audran, R., Gander, B., Corradin, G., 1997. Induction of a cytotoxic T lymphocyte response by immunization with a malaria specific CTL peptide entrapped in biodegradable polymer microspheres. Vaccine 15, 1405-1412.

Men, Y., Thomasin, C., Merkle, H.P., Gander, B., Corradin, G., 1995. A single administration of tetanus toxoid in biodegradable microspheres elicits T-cell and antibody responses similar or superior to those obtained with aluminium hydroxide. Vaccine 13, 683-689.

Mercer, J., Schelhaas, M., Helenius, A., 2010. Virus Entry by Endocytosis. Annual Review of Biochemistry, Vol 79 79, 803-833.

O'Hagan, D.T., Fox, C.B., 2015. New generation adjuvants - From empiricism to rational design. Vaccine 33, B14-B20.

Okada, C., Akbar, F., Horiike, N., Onji, M., 2005. Early development of primary biliary cirrhosis in female C57BL/6 mice because of poly I : C administration. Liver International 25, 595-603.

Ospelt, C., Brentano, F., Rengel, Y., Stanczyk, J., Kolling, C., Tak, P.P., Gay, R.E., Gay, S., Kyburz, D., 2008. Overexpression of Toll-like Receptors 3 and 4 in Synovial Tissue 
From Patients With Early Rheumatoid Arthritis Toll-like Receptor Expression in Early and Longstanding Arthritis. Arthritis and Rheumatism 58, 3684-3692.

Pasche, S., De Paul, S.M., Voros, J., Spencer, N.D., Textor, M., 2003. Poly(L-lysine)-graftpoly(ethylene glycol) assembled monolayers on niobium oxide surfaces: A quantitative study of the influence of polymer interfacial architecture on resistance to protein adsorption by ToF-SIMS and in situ OWLS. Langmuir 19, 9216-9225.

Patole, P.S., Grone, H.J., Segerer, S., Ciubar, R., Belemezova, E., Henger, A., Kretzler, M., Schlondorff, D., Anders, H.J., 2005. Viral double-stranded RNA aggravates lupus nephritis through toll-like receptor 3 on glomerular mesangial cells and antigenpresenting cells. Journal of the American Society of Nephrology 16, 1326-1338.

Salvador, A., Igartua, M., Hernandez, R.M., Pedraz, J.L., 2012. Combination of immune stimulating adjuvants with poly(lactide-co-glycolide) microspheres enhances the immune response of vaccines. Vaccine 30, 589-596.

Sharp, F.A., Ruane, D., Claass, B., Creagh, E., Harris, J., Malyala, P., Singh, M., O'Hagan, D.T., Petrilli, V., Tschopp, J., O'Neill, L.A.J., Lavelle, E.C., 2009. Uptake of particulate vaccine adjuvants by dendritic cells activates the NALP3 inflammasome. Proceedings of the National Academy of Sciences of the United States of America 106, 870-875.

Silva, A.L., Soema P.C., Slütter B., Ossendorp F., Jiskoot W. 2016. PLGA particulate delivery systems for subunit vaccines: linking particle properties to immunogenicity, Human Vaccines \& Immunotherapeutics, DOI:10.1080/21645515.2015.1117714

Singh, M., Briones, M., Ott, G., O'Hagan, D., 2000. Cationic microparticles: A potent delivery system for DNA vaccines. Proceedings of the National Academy of Sciences of the United States of America 97, 811-816.

Singh, M., Ott, G., Kazzaz, J., Ugozzoli, M., Briones, M., Donnelly, J., O'Hagan, D.T., 2001. Cationic microparticles are an effective delivery system for immune stimulatory CpG DNA. Pharmaceutical Research 18, 1476-1479.

Sobel, D.O., Newsome, J., Ewel, C.H., Bellanti, J.A., Abbassi, V., Creswell, K., Blair, O., 1992. Poly-I-C induces development of diabetes mellitus in BB rat. Diabetes 41, 515520.

Steinmassl, M., Hamprecht, K., 1994. Double fluorescence analysis of human cytomegalovirus (HCMV) infected human fibroblast cultures by flow-cytometry Increase of class-I MHC expression on uninfected cells and decrease on infected cells. Archives of Virology 135, 75-87.

Suzuki, K., Mori, A., Ishii, K.J., Saito, J., Singer, D.S., Klinman, D.M., Krause, P.R., Kohn, L.D., 1999. Activation of target-tissue immune-recognition molecules by doublestranded polynucleotides. Proceedings of the National Academy of Sciences of the United States of America 96, 2285-2290. 
Thiele, L., Merkle, H.P., Walter, E., 2003. Phagocytosis and phagosomal fate of surfacemodified microparticles in dendritic cells and macrophages. Pharmaceutical Research 20, 221-228.

Umetsu, D.T., Pober, J.S., Jabara, H.H., Fiers, W., Yunis, E.J., Burakoff, S.J., Reiss, C.S., Geha, R.S., 1985. Human dermal fibroblasts present tetanus toxoid antigen to antigenspecific T-cell clones. Journal of Clinical Investigation 76, 254-260.

Waeckerle-Men, Y., Groettrup, M., 2005. PLGA microspheres for improved antigen delivery to dendritic cells as cellular vaccines. Advanced Drug Delivery Reviews 57, 475-482.

Waeckerle-Men, Y., Scandella, E., Allmen, E.U., Ludewig, B., Gillessen, S., Merkle, H.P., Gander, B., Groettrup, M., 2004. Phenotype and functional analysis of human monocyte-derived dendritic cells loaded with biodegradable poly(lactide-co-glycolide) microspheres for immunotherapy. Journal of Immunological Methods 287, 109-124.

Waeckerle-Men, Y., Uetz-von Allmen, E., Gander, B., Scandella, E., Schlosser, E., Schmidtke, G., Merkle, H.R., Groettrup, M., 2006. Encapsulation of proteins and peptides into biodegradable poly(D,L-lactide-co-glycolide) microspheres prolongs and enhances antigen presentation by human dendritic cells. Vaccine 24, 1847-1857.

Walter, E., Merkle, H.P., 2002. Microparticle-mediated transfection of non-phagocytic cells in vitro. Journal of Drug Targeting 10, 11-21.

Wattendorf, U., Koch, M.C., Walter, E., Voeroes, J., Textor, M., Merkle, H.P., 2006. Phagocytosis of poly(L-lysine)-graft-poly(ethylene glycol) coated microspheres by antigen presenting cells: Impact of grafting ratio and poly(ethylene glycol) chain length on cellular recognition. Biointerphases 1, 123-133.

Wattendorf, U., Kreft, O., Textor, M., Sukhorukov, G.B., Merkle, H.P., 2008. Stable stealth function for hollow polyelectrolyte microcapsules through a poly(ethylene glycol) grafted polyelectrolyte adlayer. Biomacromolecules 9, 100-108.

Weck, M.M., Gruenebach, F., Werth, D., Sinzger, C., Bringmann, A., Brossart, P., 2007. TLR ligands differentially affect uptake and presentation of cellular antigens. Blood 109, 3890-3894.

Wischke, C., Zimmermann, J., Wessinger, B., Schendler, A., Borchert, H.-H., Peters, J.H., Nesselhut, T., Lorenzen, D.R., 2009. Poly(I:C) coated PLGA microparticles induce dendritic cell maturation. International Journal of Pharmaceutics 365, 61-68. 